\title{
Electroweak corrections in a pseudo Nambu-Goldstone Dark Matter model revisited
}

\author{
Seraina Glaus, ${ }^{a, b}$ Margarete Mühlleitner, ${ }^{a}$ Jonas Müller, ${ }^{a}$ Shruti Patel, ${ }^{a, b}$ \\ Tizian Römer $^{a}$ and Rui Santos ${ }^{c, d}$ \\ ${ }^{a}$ Institute for Theoretical Physics, Karlsruhe Institute of Technology, \\ 76128 Karlsruhe, Germany \\ ${ }^{b}$ Institute for Nuclear Physics, Karlsruhe Institute of Technology, \\ 76344 Karlsruhe, Germany \\ ${ }^{c}$ Centro de Física Teórica e Computacional, Faculdade de Ciências, \\ Universidade de Lisboa, \\ Campo Grande, Edifício C8 1749-016 Lisboa, Portugal \\ ${ }^{d}$ ISEL - Instituto Superior de Engenharia de Lisboa, Instituto Politécnico de Lisboa, \\ 1959-007 Lisboa, Portugal \\ E-mail: seraina.glaus@kit.edu, milada.muehlleitner@kit.edu, \\ jonas.mueller@kit.edu, shruti.patel@kit.edu, \\ tizian.roemer@student.kit.edu, rasantos@fc.ul.pt
}

ABSTRACT: Having so far only indirect evidence for the existence of Dark Matter a plethora of experiments aims at direct detection of Dark Matter through the scattering of Dark Matter particles off atomic nuclei. For the correct interpretation and identification of the underlying nature of the Dark Matter constituents higher-order corrections to the cross section of Dark Matter-nucleon scattering are important, in particular in models where the tree-level cross section is negligibly small. In this work we revisit the electroweak corrections to the dark matter-nucleon scattering cross section in a model with a pseudo Nambu-Goldstone boson as the Dark Matter candidate. Two calculations that already exist in the literature, apply different approaches resulting in different final results for the cross section in some regions of the parameter space leading us to redo the calculation and analyse the two approaches to clarify the situation. We furthermore update the experimental constraints and examine the regions of the parameter space where the cross section is above the neutrino floor but which can only be probed in the far future.

Keywords: Beyond Standard Model, Higgs Physics

ARXiv EPrint: 2008.12985 


\section{Contents}

1 Introduction 1

2 The model 2

3 Renormalisation of the PNGDM 4

3.1 Gauge sector 4

3.2 Scalar sector 5

3.3 Quark sector 7

$\begin{array}{lll}3.4 & \text { Renormalisation of the mixing angle } & 7\end{array}$

3.5 Renormalisation of the singlet VEV $v_{s} \quad 8$

4 Spin-independent cross section in the PNGDM 8

$\begin{array}{lll}4.1 & \text { SI cross section at tree level } & 10\end{array}$

$\begin{array}{lll}4.2 & \text { EW corrections to the SI cross section } & 10\end{array}$

$\begin{array}{lll}\text { 4.2.1 } & \text { Mediator corrections } & 11\end{array}$

$\begin{array}{lll}\text { 4.2.2 Upper vertex (upV) corrections } & 12\end{array}$

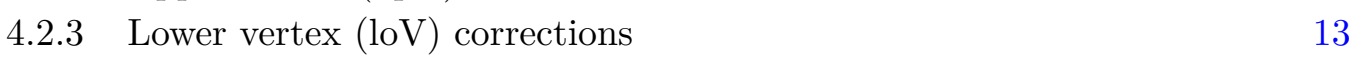

$\begin{array}{lll}\text { 4.2.4 Box diagrams } & 14\end{array}$

$\begin{array}{ll}\text { 4.2.5 General mapping to the Wilson coefficients } & 16\end{array}$

$\begin{array}{ll}\text { 4.2.6 Gluon contributions } & 17\end{array}$

5 Numerical results $\quad 19$

$\begin{array}{lll}5.1 & \text { Numerical set-up and parameters } & 19\end{array}$

$\begin{array}{lll}5.2 & \text { Results and discussion } & 21\end{array}$

6 Conclusions $\quad 26$

\section{Introduction}

Ever since Dark Matter (DM) became an inevitable ingredient in model building, all kind of proposals integrating DM candidates into phenomenologically viable models have emerged, from the simplest extensions of the Standard Model (SM) to fairly intricate models. The accumulated data from astrophysics and cosmology strongly suggests that if DM is a particle, it is most probably cold and with a mass close to the electroweak scale. Particles with these features are known as Weakly Interacting Massive Particles (WIMPs). In this work we study a simple extension of the SM where a complex singlet is added to the SM field content. The model is built such that after spontaneous symmetry breaking one of the singlet components will mix with the SM-like Higgs boson while the other one will play the role of the DM candidate. This type of extension was first proposed in refs. [1-4], reviewed with updated experimental constraints in ref. [5] and more recently in ref. [6]. 
A particular version of this extension known as the Pseudo Nambu-Goldstone DM model (PNGDM) has a scalar potential invariant under a global U(1) symmetry which would give rise to a Nambu-Goldstone boson. The symmetry is then softly broken and a pseudo Nambu-Goldstone boson emerges as the Dark Matter candidate. As discussed in previous works $[7,8]$, the nature of this particle makes the DM-nucleon tree-level cross section proportional to the velocity of the DM particle and therefore negligible (see also ref. [9] for an interesting discussion on the subject of Goldstone and pseudo-Goldstone bosons). Hence, the leading order cross section is given by its one-loop contribution. The first calculation of the electroweak corrections to this process was performed in ref. [8] and almost at the same time a second calculation appeared in ref. [10]. In this work we will perform once again the calculation of the electroweak corrections to the DM-nucleon cross section while discussing in detail the main differences with respect to the two previous calculations and the reasons for settling this issue. Our calculation will be performed with a different renormalisation scheme. This allows us to perform a rough estimate of the remaining uncertainties on the cross section due to missing higher-order corrections.

We then perform a scan in the parameter space taking into account the most relevant theoretical and experimental constraints. We will show that there are still allowed points in the parameter space above the neutrino floor [11] but only experiments in the far future will be able to probe them.

The paper is organized as follows. In section 2 we briefly present the complex singlet extension of the SM while in section 3 we introduce the renormalisation of the model. The various aspects of the DM direct-detection cross section at tree level and at one-loop level are discussed in 4 . In section 5 we discuss the results and future prospects of DM detection in this model taking into account the most recent constraints. We summarise our findings in section 6 .

\section{The model}

A simple extension of the SM by a scalar gauge singlet is enough to provide a valid DM candidate. The new complex scalar field $S$, with zero hypercharge and zero isospin only enters the model via the scalar potential that can be written as

$$
V=-\frac{\mu_{H}^{2}}{2}|H|^{2}+\frac{\lambda_{H}}{2}|H|^{4}-\frac{\mu_{S}^{2}}{2}|S|^{2}+\frac{\lambda_{S}}{2}|S|^{4}+\lambda_{H S}|H|^{2}|S|^{2}-\frac{m_{\chi}^{2}}{4}\left(S^{2}+S^{* 2}\right),
$$

where the mass parameters $\mu_{H}^{2}, \mu_{S}^{2}, m_{\chi}^{2}$ and the quartic couplings $\lambda_{S}, \lambda_{H}, \lambda_{H S}$ are real due to hermicity. The doublet $H$ and singlet $S$ fields are expanded as follows

$$
H=\left(\begin{array}{c}
G^{+} \\
\frac{1}{\sqrt{2}}\left(v+\Phi_{H}+i G^{0}\right)
\end{array}\right), \quad S=\frac{1}{\sqrt{2}}\left(v_{s}+\Phi_{S}+i \chi\right),
$$

with the electroweak vacuum expectation value (VEV) $v$ and the singlet VEV $v_{s}$. With this definition the model is invariant under the DM charge conjugation $S \rightarrow S^{*}$, which guarantees the stability of the imaginary part of $S$. Furthermore, in order to simplify the potential, an invariance under the $Z_{2}$ symmetry $S \rightarrow-S$ has also been imposed. The 
real part of $S$ develops a vacuum expectation value (VEV), while the doublet develops the usual (SM) VEV that gives mass to the SM fermions and gauge bosons,

$$
\langle H\rangle=\frac{1}{\sqrt{2}}\left(\begin{array}{l}
0 \\
v
\end{array}\right), \quad\langle S\rangle=\frac{v_{s}}{\sqrt{2}}
$$

with $v=2 m_{W} / g, m_{W}$ the $W$ boson mass and $g$ the $\mathrm{SU}(2)$ coupling constant. Because the real part of $S$ acquires a $\mathrm{VEV}, \Phi_{S}$ cannot be a viable DM candidate and it mixes with the doublet real neutral component $\Phi_{H}$. Using the minimum conditions

$$
\begin{array}{lll}
\left.\frac{\partial V}{\partial H}\right|_{\mathrm{VEV}}=0 & \Longleftrightarrow & T_{H} \equiv \frac{v}{2}\left(-\mu_{H}^{2}+\lambda_{H} v^{2}+\lambda_{H S} v_{s}^{2}\right)=0, \\
\left.\frac{\partial V}{\partial S}\right|_{\mathrm{VEV}}=0 & \Longleftrightarrow & T_{S} \equiv \frac{v_{s}}{2}\left(-\mu_{S}^{2}+\lambda_{S} v_{s}^{2}+\lambda_{H S} v^{2}-m_{\chi}^{2}\right)=0 .
\end{array}
$$

we can write the mass matrix of the two neutral states as

$$
\mathcal{M}_{T}^{2}=\mathcal{M}^{2}+\mathcal{T}, \quad \mathcal{M}^{2}=\left(\begin{array}{cc}
\lambda_{H} v^{2} & \lambda_{H S} v v_{s} \\
\lambda_{H S} v v_{s} & \lambda_{S} v_{s}^{2}
\end{array}\right), \quad \mathcal{T}=\left(\begin{array}{cc}
T_{H} / v & 0 \\
0 & T_{S} / v_{s}
\end{array}\right),
$$

The mass eigenstates $h_{1}$ and $h_{2}$ are obtained from the gauge eigenstates via

$$
\left(\begin{array}{l}
h_{1} \\
h_{2}
\end{array}\right) \equiv R(\alpha)\left(\begin{array}{l}
\Phi_{H} \\
\Phi_{S}
\end{array}\right), \quad M^{2} \equiv R(\alpha) \mathcal{M}^{2} R^{-1}(\alpha)=\left(\begin{array}{cc}
m_{h_{1}}^{2} & 0 \\
0 & m_{h_{2}}^{2}
\end{array}\right)
$$

with the orthogonal matrix $R(\alpha)$

$$
R(\alpha) \equiv\left(\begin{array}{cc}
\cos \alpha & \sin \alpha \\
-\sin \alpha & \cos \alpha
\end{array}\right)
$$

One of these mass eigenstates is identified as the $125 \mathrm{GeV}$ Higgs boson. The DM particle is given by $\chi$. Exploiting the tadpole conditions eq. (2.4) its mass can be written as

$$
m_{\chi}^{2}+\frac{T_{S}}{v_{S}}
$$

We also require that the potential is bounded from below inducing the tree-level conditions

$$
\lambda_{H}>0, \quad \lambda_{S}>0, \quad \lambda_{H S}>-\sqrt{\lambda_{H} \lambda_{S}} .
$$

The parameters of the potential can be written as functions of the masses, mixing angle and the VEVs as

$$
\begin{aligned}
\lambda_{H S} & =-\frac{m_{h_{2}}^{2}-m_{h_{1}}^{2}}{2 v v_{s}} \sin 2 \alpha, \\
\lambda_{H} & =\frac{m_{h_{2}}^{2} \sin ^{2} \alpha+m_{h_{1}}^{2} \cos ^{2} \alpha}{v^{2}}, \\
\lambda_{S} & =\frac{m_{h_{2}}^{2} \cos ^{2} \alpha+m_{h_{1}}^{2} \sin ^{2} \alpha}{v_{s}^{2}} .
\end{aligned}
$$


We choose the following parameters as independent input parameters,

$$
v, v_{s}, \alpha, m_{h_{1}}^{2}, m_{h_{2}}^{2}, m_{\chi}^{2}, T_{H}, T_{S} .
$$

Some final comments regarding the scalar potential are in order. The potential is invariant under a $\mathrm{U}(1)$ symmetry $\left(S \rightarrow e^{i \alpha} S\right)$ that is softly broken by the dimension-two term proportional to $m_{\chi}^{2}$. The Goldstone boson related to the $\mathrm{U}(1)$ symmetry acquires a mass proportional to $m_{\chi}^{2}$. Due to the $Z_{2}$ symmetry there are no more terms contributing to the mass of the pseudo Nambu-Goldstone boson. Hence, the U(1) symmetry is recovered by setting $m_{\chi}^{2}=0$, where the true Nambu-Goldstone boson is recovered.

\section{Renormalisation of the PNGDM}

In the following, we present the renormalisation of the PNGDM in order to be able to calculate the electroweak (EW) corrections to the scattering process of the pseudo NambuGoldstone DM particle with a nucleon. Having defined the full set of input parameters in eq. (2.11), the bare parameters $p_{0}$ are replaced with the renormalized ones, $p$, according to

$$
p_{0}=p+\delta p,
$$

where $\delta p$ corresponds to the counterterm of the respective bare parameter $p_{0}$. For a generic bare field $\Psi_{0}$ (scalar, fermion or vector field), the renormalized field $\Psi$ is expressed as

$$
\Psi_{0}=\sqrt{Z_{\Psi}} \Psi
$$

with the field strength renormalisation constant $Z_{\Psi} \equiv 1+\delta Z_{\Psi}$. Note that $Z_{\Psi}$ is a matrix for mixing fields as present in the PNGDM. Dropping for simplicity the index 0 in the following, we hence make the following replacements

$$
\begin{aligned}
p & \rightarrow p+\delta p \\
\Psi & \rightarrow\left(1+\frac{1}{2} \delta Z_{\Psi}\right) \Psi,
\end{aligned}
$$

and analogously for the tadpole parameter

$$
T \rightarrow T+\delta T .
$$

In the following we discuss each sector separately.

\subsection{Gauge sector}

The gauge sector of the PNGDM is not extended compared to the SM. To set our notation and conventions we will list the counterterms of the gauge sector in the following. We choose to perform the renormalisation in the mass basis of the PNGDM, so that the following set of on-shell (OS) counterterms are taken for the gauge sector

$$
\begin{aligned}
m_{W}^{2} & \rightarrow m_{W}^{2}+\delta m_{W}^{2}, \\
m_{Z}^{2} & \rightarrow m_{Z}^{2}+\delta m_{Z}^{2}, \\
e & \rightarrow e+\delta Z_{e} e, \\
g & \rightarrow g+\delta g
\end{aligned}
$$


where $m_{Z}$ is the mass of the EW neutral gauge boson $Z$ and the electric coupling is denoted by $e$. The renormalized fields are obtained through the field strength renormalisation constants as

$$
\begin{aligned}
& W^{ \pm} \rightarrow\left(1+\frac{1}{2} \delta Z_{W W}\right) W^{ \pm} \\
& \left(\begin{array}{l}
Z \\
\gamma
\end{array}\right) \rightarrow\left(\begin{array}{cc}
1+\frac{1}{2} \delta Z_{Z Z} & \frac{1}{2} \delta Z_{Z \gamma} \\
\frac{1}{2} \delta Z_{\gamma Z} & 1+\frac{1}{2} \delta Z_{\gamma \gamma}
\end{array}\right)\left(\begin{array}{l}
Z \\
\gamma
\end{array}\right)
\end{aligned}
$$

Applying OS conditions yields the following mass counterterms

$$
\delta m_{W}^{2}=\operatorname{Re} \Sigma_{W W}^{T}\left(m_{W}^{2}\right) \quad \text { and } \quad \delta m_{W}^{2}=\operatorname{Re} \Sigma_{Z Z}^{T}\left(m_{Z}^{2}\right)
$$

with $T$ indicating the transverse part of the self-energies $\Sigma_{i i}(i i=W, Z)$. The counterterm for the gauge coupling $g$ is obtained from the one for the electric charge and the one for the Weinberg angle $\theta_{W}$ using

$$
e=g \sin \theta_{W}, \quad \text { with } \quad \cos \theta_{W}=\frac{m_{W}}{m_{Z}} .
$$

The electric charge counterterm is fixed in the Thomson limit, which by making use of Ward identities allows us to write $[12]^{1}$

$$
\delta Z_{e}=\left.\frac{1}{2} \frac{\partial \Sigma_{\gamma \gamma}^{T}\left(p^{2}\right)}{\partial p^{2}}\right|_{p^{2}=0}+\frac{s_{W}}{c_{W}} \frac{\Sigma_{\gamma Z}^{T}(0)}{m_{Z}^{2}},
$$

where we introduced the short-hand notation $s_{W} \equiv \sin \theta_{W}, c_{W} \equiv \cos \theta_{W}$, and

$$
\frac{\delta g}{g}=\delta Z_{e}+\frac{1}{2} \frac{1}{m_{Z}^{2}-m_{W}^{2}}\left(\delta m_{W}^{2}-c_{W}^{2} \delta m_{Z}^{2}\right)
$$

The corresponding wave-function renormalisation constants guaranteeing the correct OS properties are given by

$$
\begin{aligned}
& \delta Z_{W W}=-\left.\operatorname{Re} \frac{\partial \Sigma_{W W}^{2}\left(p^{2}\right)}{\partial p^{2}}\right|_{p^{2}=m_{W}^{2}}, \\
&\left(\begin{array}{ll}
\delta Z_{Z Z} & \delta Z_{Z \gamma} \\
\delta Z_{\gamma Z} & \delta Z_{\gamma \gamma}
\end{array}\right)=\left(\begin{array}{cc}
-\left.\operatorname{Re} \frac{\partial \Sigma_{Z Z}^{T}\left(p^{2}\right)}{\partial p^{2}}\right|_{p^{2}=m_{Z}^{2}} & 2 \frac{\Sigma_{Z \gamma}^{T}(0)}{m_{Z}^{2}} \\
-2 \frac{\Sigma_{Z \gamma}^{T}(0)}{m_{Z}^{2}} & -\left.\operatorname{Re} \frac{\partial \Sigma_{\gamma \gamma}^{T}\left(p^{2}\right)}{\partial p^{2}}\right|_{p^{2}=0}
\end{array}\right) .
\end{aligned}
$$

\subsection{Scalar sector}

In the PNGDM we have two additional scalars, one extra CP-even Higgs boson and the DM candidate $\chi$. The two CP-even scalars are mass-ordered as $h_{1}$ and $h_{2}$ with $m_{h_{1}}<m_{h_{2}}$

\footnotetext{
${ }^{1}$ Note that the sign in the second term of eq. (3.10) differs from the one in [12] due to different sign conventions in the covariant derivative.
} 
and the SM-like Higgs boson, with a mass of $125.09 \mathrm{GeV}$, can be either of them. We again use an OS scheme for the fields. The field strength renormalisation constants read

$$
\left(\begin{array}{l}
h_{1} \\
h_{2}
\end{array}\right) \rightarrow\left(\begin{array}{cc}
1+\frac{1}{2} \delta Z_{h_{1} h_{1}} & \frac{1}{2} \delta Z_{h_{1} h_{2}} \\
\frac{1}{2} \delta Z_{h_{2} h_{1}} & 1+\frac{1}{2} \delta Z_{h_{2} h_{2}}
\end{array}\right)\left(\begin{array}{l}
h_{1} \\
h_{2}
\end{array}\right) .
$$

The mass matrix with the additional tadpole contributions is given by

$$
\mathcal{M}_{h_{1} h_{2}}=\left(\begin{array}{cc}
m_{h_{1}}^{2} & 0 \\
0 & m_{h_{2}}^{2}
\end{array}\right)+\underbrace{R(\alpha)\left(\begin{array}{cc}
T_{H} / v & 0 \\
0 & T_{S} / v_{S}
\end{array}\right) R(\alpha)^{T}}_{\equiv \delta T} .
$$

The rotation matrix $R(\alpha)$ is defined in eq. (2.7) and diagonalises the gauge eigenstates in the Higgs mass basis. The tadpole terms $\delta T$ in the tree-level mass matrix are bare parameters. At next-to-leading (NLO) they get shifted due to EW corrections to the vaccuum state of the potential. Defining the tree-level VEV to be the same to all orders of perturbation theory, requires the introduction of tadpole counterterms such that the one-loop renormalized one-point function $\hat{T}_{i}(i=H, S)$ vanishes

$$
\hat{T}_{i}=T_{i}-\delta T_{i} \stackrel{!}{=} 0 .
$$

Note that the rotation matrix from the gauge states to the Higgs mass states also applies to the tadpoles, yielding the relation between the tadpoles $T_{i}(i=H, S)$ and $T_{h_{i}}(i=1,2)$

$$
\left(\begin{array}{c}
T_{h_{1}} \\
T_{h_{2}}
\end{array}\right)=R(\alpha) \cdot\left(\begin{array}{c}
T_{H} \\
T_{S}
\end{array}\right)
$$

The one-loop mass counterterm of the Higgs sector is then given by

$$
\mathcal{M}_{h_{1} h_{2}} \rightarrow \mathcal{M}_{h_{1} h_{2}}+\delta \mathcal{M}_{h_{1} h_{2}}
$$

with

$\delta \mathcal{M}_{h_{1} h_{2}}=\left(\begin{array}{cc}\delta m_{h_{1}}^{2} & 0 \\ 0 & \delta m_{h_{2}}^{2}\end{array}\right)+R(\alpha)\left(\begin{array}{cc}\frac{\delta T_{H}}{v} & 0 \\ 0 & \frac{\delta T_{S}}{v_{S}}\end{array}\right) R(\alpha)^{T} \equiv\left(\begin{array}{cc}\delta m_{h_{1}}^{2} & 0 \\ 0 & \delta m_{h_{2}}^{2}\end{array}\right)+\left(\begin{array}{cc}\delta T_{h_{1} h_{1}} & \delta T_{h_{1} h_{2}} \\ \delta T_{h_{2} h_{1}} & \delta T_{h_{2} h_{2}}\end{array}\right)$.

Equation (3.18) is strictly expanded to one-loop order, so that terms $\mathcal{O}\left(\delta \alpha \delta T_{i}\right)$ are dropped. Applying OS conditions yields $(i=1,2)$

$$
\begin{aligned}
\delta m_{h_{i}}^{2} & =\operatorname{Re}\left[\Sigma_{h_{i} h_{i}}\left(m_{h_{i}}^{2}\right)-\delta T_{h_{i} h_{i}}\right], \\
\delta Z_{h_{i} h_{i}} & =-\operatorname{Re}\left[\frac{\partial \Sigma_{h_{i} h_{i}}\left(p^{2}\right)}{\partial p^{2}}\right]_{p^{2}=m_{h_{i}}^{2}}, \\
\delta Z_{h_{i} h_{j}} & =\frac{2}{m_{h_{i}}^{2}-m_{h_{j}}^{2}} \operatorname{Re}\left[\Sigma_{h_{i} h_{j}}\left(m_{h_{j}}^{2}\right)-\delta T_{h_{i} h_{j}}\right], \quad i \neq j .
\end{aligned}
$$

There is just one DM candidate and therefore the renormalisation constants are defined by

$$
\delta Z_{\chi \chi}=-\left.\operatorname{Re}\left[\frac{\partial \Sigma_{\chi \chi}\left(p^{2}\right)}{\partial p^{2}}\right]\right|_{p=m_{\chi}^{2}}, \quad \delta m_{\chi}^{2}=\operatorname{Re}\left[\Sigma_{\chi \chi}\left(p^{2}=m_{\chi}^{2}\right)-\frac{\delta T_{S}}{v_{S}}\right],
$$

with the self-energy $\Sigma_{\chi \chi}$ of the DM candidate. 


\subsection{Quark sector}

In the quark sector we assume a diagonal CKM matrix for simplicity. This means we neglect flavor mixing and the OS scheme is applied for each quark individually. The field strength renormalisation constant has to be formulated for the left- and right-handed field of the quarks $(q=u, d, s, b, t)[13]$

$$
q_{L / R} \rightarrow\left(1+\frac{1}{2} \delta Z_{q q}^{L / R}\right) q
$$

and the mass counterterm is introduced through

$$
m_{q} \rightarrow m_{q}+\delta m_{q}
$$

The two-point correlation function of the quarks is written as

$$
\Gamma_{q q}(p)=i\left(\not p-m_{q}\right)+i\left[\not p \omega_{-} \Sigma_{q q}^{L}\left(p^{2}\right)+\not p \omega_{+} \Sigma_{q q}^{R}\left(p^{2}\right)+m_{q}\left(\omega_{+}+\omega_{-}\right) \Sigma_{q q}^{S}\left(p^{2}\right)\right],
$$

where the superscripts $L, R$ and $S$ correspond to the left-, right-handed and scalar parts of the self-energies, respectively. The $\omega_{ \pm}$are the left- and right-handed projectors. The full set of counterterms is then given in terms of the left-/right-handed and scalar parts of the respective self-energies as

$$
\begin{aligned}
\delta m_{q} & =\frac{m_{q}}{2} \operatorname{Re}\left[\Sigma_{q q}^{L}\left(m_{q}^{2}\right)+\Sigma_{q q}^{R}\left(m_{q}^{2}\right)+2 \Sigma_{q q}^{S}\left(m_{q}^{2}\right)\right] \\
\delta Z_{q q}^{L} & =-\operatorname{Re}\left[\Sigma_{q q}^{L}\left(m_{q}^{2}\right)\right]-\left.m_{q}^{2} \operatorname{Re}\left[\frac{\partial \Sigma_{q q}^{L}\left(p^{2}\right)}{\partial p^{2}}+\frac{\partial \Sigma_{q q}^{R}\left(p^{2}\right)}{\partial p^{2}}+2 \frac{\partial \Sigma_{q q}^{S}\left(p^{2}\right)}{\partial p^{2}}\right]\right|_{p^{2}=m_{q}^{2}}, \\
\delta Z_{q q}^{R} & =-\operatorname{Re}\left[\Sigma_{q q}^{R}\left(m_{q}^{2}\right)\right]-\left.m_{q}^{2} \operatorname{Re}\left[\frac{\partial \Sigma_{q q}^{L}\left(p^{2}\right)}{\partial p^{2}}+\frac{\partial \Sigma_{q q}^{R}\left(p^{2}\right)}{\partial p^{2}}+2 \frac{\partial \Sigma_{q q}^{S}\left(p^{2}\right)}{\partial p^{2}}\right]\right|_{p^{2}=m_{q}^{2}} .
\end{aligned}
$$

\subsection{Renormalisation of the mixing angle}

The rotation eq. (2.6) of the interaction states $\Phi_{H}$ and $\Phi_{S}$ to the mass eigenstates $h_{1}$ and $h_{2}$ introduces the mixing angle $\alpha$ that needs to be renormalized as well. The renormalisation of the mixing angles in SM extensions was thoroughly discussed in refs. [14-26]. There are many possibilities to renormalize the mixing angle. One possibility is to use a physical process, like a decay. However, it is known that the usage of a process-dependent scheme may yield an unphysically large counterterm [15] which in turn leads to extremely large corrections. In this work we will use the KOSY scheme, proposed in refs. [27, 28], which connects the angle counterterm with the usual OS counterterms for the scalar. ${ }^{2}$ The bare parameter $\alpha_{0}$ can be expressed in terms of the renormalized one, $\alpha$, as

$$
\alpha_{0}=\alpha+\delta \alpha .
$$

Considering the field strength renormalisation before the rotation,

$$
\left(\begin{array}{l}
h_{1} \\
h_{2}
\end{array}\right)=R(\alpha+\delta \alpha) \sqrt{Z_{\Phi}}\left(\begin{array}{l}
\Phi_{H} \\
\Phi_{S}
\end{array}\right),
$$

\footnotetext{
${ }^{2}$ Note, however, that the KOSY scheme can lead to gauge-dependent results [15].
} 
and expanding it to strict one-loop order,

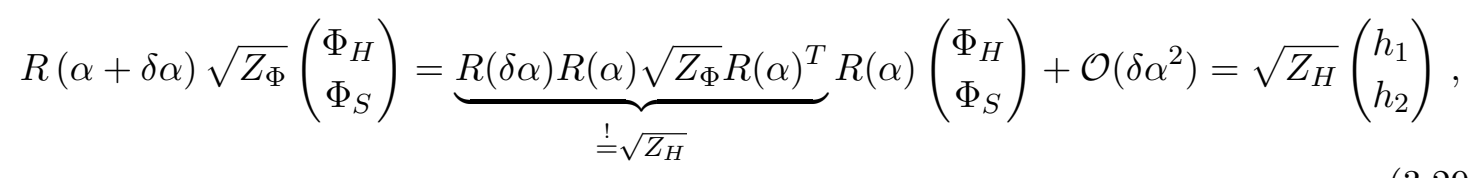

yields the field strength renormalisation matrix $\sqrt{Z_{H}}$ connecting the bare and renormalised fields in the mass basis. Using the rotation matrix expanded at one-loop order results in

$$
\sqrt{Z_{H}}=R(\delta \alpha)\left(\begin{array}{cc}
1+\frac{\delta Z_{h_{1} h_{1}}}{2} & \delta C_{h} \\
\delta C_{h} & 1+\frac{\delta Z_{h_{2} h_{2}}}{2}
\end{array}\right) \approx\left(\begin{array}{cc}
1+\frac{\delta Z_{h_{1} h_{1}}}{2} & \delta C_{h}+\delta \alpha \\
\delta C_{h}-\delta \alpha & 1+\frac{\delta Z_{h_{2} h_{2}}}{2}
\end{array}\right) .
$$

Demanding that the field mixing vanishes on the mass shell is equivalent to identifying the off-diagonal elements of $\sqrt{Z_{H}}$ with those in eq. (3.13),

$$
\frac{\delta Z_{h_{1} h_{2}}}{2} \stackrel{!}{=} \delta C_{h}+\delta \alpha \quad \text { and } \quad \frac{\delta Z_{h_{2} h_{1}}}{2} \stackrel{!}{=} \delta C_{h}-\delta \alpha
$$

With eq. (3.21) the mixing angle counterterm reads

$$
\begin{aligned}
\delta \alpha & =\frac{1}{4}\left(\delta Z_{h_{1} h_{2}}-\delta Z_{h_{2} h_{1}}\right) \\
& =\frac{1}{2\left(m_{h_{1}}^{2}-m_{h_{2}}^{2}\right)} \operatorname{Re}\left(\Sigma_{h_{1} h_{2}}\left(m_{h_{1}}^{2}\right)+\Sigma_{h_{1} h_{2}}\left(m_{h_{2}}^{2}\right)-2 \delta T_{h_{1} h_{2}}\right) .
\end{aligned}
$$

We do not perform a comparison of various renormalisation schemes, like a processdependent, $\overline{\mathrm{MS}}$, or the KOSY scheme, in this work. We note, however, that in our previous work [29], when comparing these three schemes, we found that only the KOSY scheme led to reasonable NLO predictions.

\subsection{Renormalisation of the singlet VEV $v_{s}$}

In the Standard Tadpole (ST) scheme that we are using in this work, there is no need to renormalize the singlet VEV $v_{s}$. It was shown in ref. [30] that when choosing an $R_{\xi}$ gauge in the ST scheme there is no divergence associated with $v_{s}$ at one-loop order if the scalar field obeys a rigid invariance. In these SM extensions the singlet field is disconnected from the gauge sector and hence invariant under global gauge transformations. This is exactly the case for typical extended scalar sectors with a singlet field, like the complex (or real) singlet extension of the SM or the Next-to-2-Higgs-Doublet Model, where a real singlet field is added to the 2-Higgs-Doublet Model. We note, however, that in the alternative tadpole scheme as defined in ref. [31] for the SM and in ref. [18] for the N2HDM this is no longer true and a counterterm for $v_{s}$ is needed.

\section{Spin-independent cross section in the PNGDM}

In the following, the calculation of the spin-independent (SI) cross section for the direct detection of DM is presented. The starting point is the scattering process of a DM particle 
with the nucleon. The effective coupling of this process is denoted by $\alpha_{n}$,

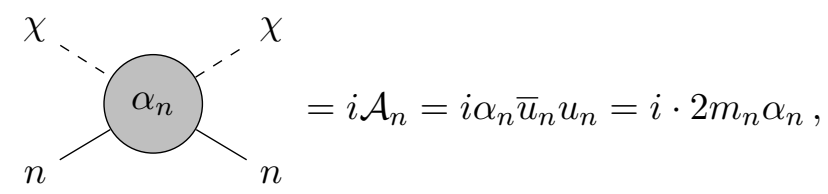

where it is additionally assumed that the momentum of the nucleon is not altered, that is, the momentum transfer between the DM particle and the nucleon is negligible. We can then use the normalisation for the spinors, $\bar{u}_{n} u_{n}=2 m_{n}$. The DM-nucleon cross section with the interaction in eq. (4.1) is then given by

$$
\sigma_{n}=\frac{1}{4 \pi}\left(\frac{m_{n}}{m_{n}+m_{\chi}}\right)^{2}\left|\alpha_{n}\right|^{2},
$$

where $m_{n}$ corresponds to the nucleon mass and $m_{\chi}$ to the DM mass. Since the nucleon is a bound state the contributions to the effective DM-nucleon coupling is on the one hand given by the light valence quarks $(q=u, d, s)$ and on the other hand by the gluon interactions. In order to calculate the cross section the parton basis is used to describe the interaction between the DM and the nucleon. The SI DM-nucleon cross section is calculated by taking the related operators in the non-relativistic limit. The parton operator basis forming the most general SI-interactions for scalar DM is given by [32]

$$
\mathcal{L}_{\text {eff }}=\sum_{q} C_{S}^{q} \mathcal{O}_{S}^{q}+C_{S}^{g} \mathcal{O}_{S}^{g}+\sum_{q} C_{T}^{q} \mathcal{O}_{T}^{q}
$$

with the operators

$$
\begin{aligned}
\mathcal{O}_{S}^{q} & =m_{q} \chi^{2} \bar{q} q, \\
\mathcal{O}_{S}^{g} & =\frac{\alpha_{s}}{\pi} \chi^{2} G_{\mu \nu}^{a} G^{a \mu \nu}, \\
\mathcal{O}_{T}^{q} & =\frac{1}{m_{\chi}^{2}} \chi^{2} i \partial^{\mu} i \partial^{\nu} \underbrace{\frac{1}{2} i \bar{q}\left(\partial_{\mu} \gamma_{\nu}+\partial_{\nu} \gamma_{\mu}-\frac{1}{2} g_{\mu \nu} \not \partial\right) q}_{\equiv \mathcal{O}_{\mu \nu}^{q}} .
\end{aligned}
$$

The operators are built with the DM field $\chi$, the quark spinor $q$ and the gluon field strength tensor $G_{a}^{\mu \nu}$ and $\alpha_{s}$ denotes the strong coupling constant. The operator $\mathcal{O}_{S}^{q}$ describes the interaction induced by the quark-DM interactions and $\mathcal{O}_{S}^{g}$ the one induced by the gluon-DM interactions. The twist-2 operator $\mathcal{O}_{\mu \nu}^{q}$ also contributes to the SI cross section due to additional gluon induced interactions. Assuming on-shell nucleon states $|n\rangle$, the expectation values of the operators in eq. (4.4) can be expressed as [33-35]

$$
\begin{aligned}
\left\langle n\left|m_{q} \bar{q} q\right| n\right\rangle & \equiv m_{n} f_{q}^{n}, \\
\left\langle n\left|-\frac{\alpha_{s}}{12 \pi} G_{\mu \nu}^{a} G^{a \mu \nu}\right| n\right\rangle & \equiv \frac{2}{27} m_{n} f_{g}^{n},
\end{aligned}
$$

with the nucleon matrix elements $f_{q}^{n}$ and $f_{g}^{n}$ calculated on the lattice. The numerical values for the matrix elements used in the analysis are given in section 5.1. Eq. (4.5) allows to 
formulate the effective DM-nucleon coupling $\alpha_{n}$ in terms of the Wilson coefficients defined in eq. (4.3) arriving at [36]

$$
\sigma_{n}=\frac{1}{\pi}\left(\frac{m_{n}}{m_{\chi}+m_{n}}\right)^{2}\left|\sum_{q=u, d, s} m_{n} f_{q}^{n} C_{S}^{q}-\frac{8}{9} m_{n} f_{g}^{n} C_{S}^{g}+\frac{3}{4} m_{n} \sum_{q=u, d, s, c, b}\left(q^{n}(2)+\bar{q}^{n}(2)\right) C_{T}^{q}\right|^{2} .
$$

The numerical values of the second momenta of the quarks $q^{n}(2)$ are also given in section 5.1 .

In order to give an estimate of the DM-nucleon cross section the remaining task is to calculate the Wilson coefficients $C_{S}^{q / g}$ and $C_{T}^{q}$ in eq. (4.6).

\subsection{SI cross section at tree level}

We will start by showing that the SI cross section vanishes in the limit of vanishing momentum transfer. The Feynman diagrams representing the quark contributions together with the corresponding amplitude are given by

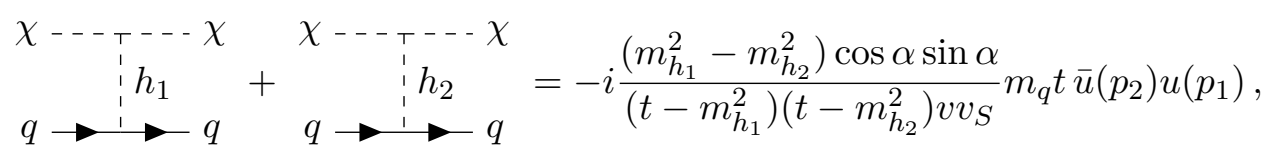

where $m_{h_{i}}(i=1,2)$ are the neutral Higgs boson masses and $t=\left(p_{\chi}-p_{q}\right)^{2}$ the Mandelstam variable. The momenta of the DM particle and the quark are denoted by $p_{\chi}$ and $p_{q}$, respectively. The amplitude in eq. (4.7) allows to read off the Wilson coefficient $C_{S}^{q}$ by identifying $m_{q} \bar{u} u$ as the operator $\mathcal{O}_{S}^{q}$. The amplitude and therefore the Wilson coefficient is proportional to the momentum transfer $t$ and vanishes in the limit of vanishing momentum transfer. Hence, the quark contribution to the SI cross section is zero. Note that this behaviour is related to the U(1) symmetry of the model as will be discussed later. Let us show that also the gluon part of the cross section vanishes in the same limit which implies that the SI cross section vanishes at tree level in the limit of vanishing momentum transfer. (Note that the twist-2 operator does not contribute at leading order.)

The QCD trace anomaly allows to relate the quark operator of the heavy quarks $Q=b, c, t$ with the gluon field strength tensor yielding the effective gluon interaction with DM particles

$$
m_{Q} \bar{Q} Q \rightarrow-\frac{\alpha_{s}}{12 \pi} G_{\mu \nu}^{a} G^{a \mu \nu}
$$

The corresponding Feynman diagram is depicted in figure 1 and can be calculated by first calculating the process with a (heavy) external quark as in eq. (4.7) and using eq. (4.8) to determine the effective gluon interaction. These amplitudes are then used to the extract the Wilson coefficients $C_{S}^{g}$. Note that the gluon contributions are extracted in the same way as in eq. (4.7), and are therefore proportional to the momentum transfer $t$. Consequently, also the gluon contributions vanish in the limit of vanishing momentum transfer.

\subsection{EW corrections to the SI cross section}

As shown in the last section, the SI cross section of the PNGDM is suppressed at tree level due to its proportionality to the momentum exchange. Since we work in the limit $t=0$, 


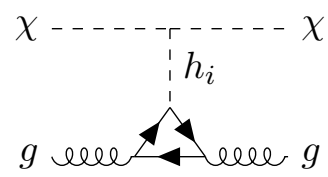

Figure 1. Interaction of a DM particle and a gluon via a Higgs boson mediator and a quark loop.

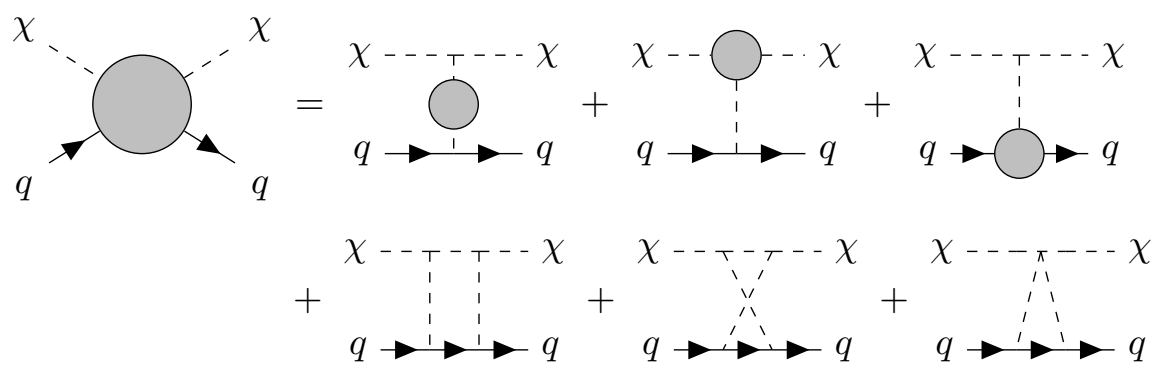

Figure 2. One-loop EW corrections to DM-quark scattering. They are given by propagator corrections, vertex corrections, box and triangle diagrams.

the tree-level cross section vanishes and we have to calculate the cross section in the next order of perturbation theory. In this section we calculate the EW corrections to the SI cross section which follows very closely our approach presented in ref. [29] and updated in ref. [37]. Note that the vector DM model presented in our previous work does not show the tree-level suppression present in the PNGDM, hence the cross section is now calculated by taking the NLO amplitude squared whereas in vector DM model the LO times NLO term was taken. The generic one-loop EW corrections are depicted in figure 2, where the gray blob denotes the renormalized 4-point vertex (left-side), the renormalized propagator corrections (first diagram on the right-hand side), the upper renormalized vertex (second diagram), the lower renormalized vertex (third diagram) and the box corrections (last three diagrams). The box corrections can be split in the genuine square box corrections, crossed box and triangle corrections. In figure 2 only the quark contributions are shown and we will comment on the gluon contributions at NLO later on.

\subsubsection{Mediator corrections}

In this section we will discuss the propagator corrections. To calculate the one-loop corrections to the mediator we first evaluate all genuine one-loop diagrams in figure 3 and construct the corresponding counterterm. This can be achieved by evaluating the renormalized one-loop propagator $(i, j=1,2)$

$$
\Delta_{h_{i} h_{j}}=-\frac{\hat{\Sigma}_{h_{i} h_{j}}\left(p^{2}=0\right)}{m_{h_{i}}^{2} m_{h_{j}}^{2}},
$$

with the renormalised self-energy matrix

$$
\left(\begin{array}{ll}
\hat{\Sigma}_{h_{1} h_{1}} & \hat{\Sigma}_{h_{1} h_{2}} \\
\hat{\Sigma}_{h_{2} h_{1}} & \hat{\Sigma}_{h_{2} h_{2}}
\end{array}\right) \equiv \hat{\Sigma}\left(p^{2}\right)=\Sigma\left(p^{2}\right)-\delta m^{2}-\delta T+\frac{\delta Z}{2}\left(p^{2}-\mathcal{M}^{2}\right)+\left(p^{2}-\mathcal{M}^{2}\right) \frac{\delta Z}{2}
$$


a)

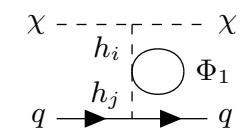

f) $\chi-\overline{h_{i}} T^{---} \chi$

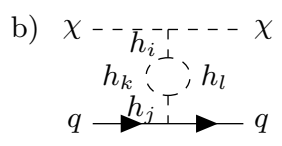

c)

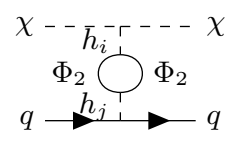

d)

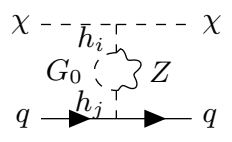

e)

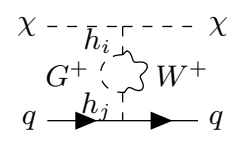

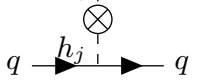

Figure 3. The one-loop EW corrections to the mediator. They can be split in the genuine one-loop diagrams a)-e)) and the respective counterterm amplitude (diagram f)). The indices $i, j, k, l=1,2$ indicates the respective Higgs mediator $h_{1}, h_{2}$. The possible field insertions are given by $\Phi_{1}=\left\{h_{i}, \chi, G^{(0, \pm)}, Z, W^{ \pm}\right\}$and $\Phi_{2}=\left\{\chi, G^{(0, \pm)}, Z, W^{ \pm}, \eta_{Z}, \eta_{W}, f\right\}$, where $f$ stands for all SM fermions, $G^{(0, \pm)}$ for the neutral and charged Goldstone bosons, respectively, and $\eta_{Z, W}$ for the ghost fields.

We now have everything to determine the contribution of the diagrams in figure 3 . Note that the field strength renormalisation constant $\delta Z$ is introduced artificially, since the Higgs bosons correspond to an internal degree of freedom. As it turns out, if the field strength renormalisation constants are included in all separate topologies (lower vertex, upper vertex and mediator corrections), they cancel each other exactly in the sum. Hence, in the end no artificially introduced $\delta Z$ parts remain in the calculation. The inclusion of these $\delta Z$ factors on the other hand allows to check for the UV finiteness in each topology by itself, simplifying the calculation or rather the bookkeeping of the contributions.

\subsubsection{Upper vertex (upV) corrections}

The upper vertex corrections - referred to as up $V$ - are depicted in figure 4. Diagrams a) to f) are the genuine one-loop corrections and are calculated in the limit of vanishing momentum transfer (i.e. the incoming momentum is equal to the outgoing momentum, $\left.p_{\text {in }}=p_{\text {out }}\right)$. Note that this specific limit is stricter than taking $q^{2}=\left(p_{\chi}-p_{q}\right)^{2}=0$ implying for instance vanishing Gram determinants complicating the reduction to the standard oneloop scalar integrals. The numerical evaluation of the integrals is performed with the Collier package [38-40] and explicitly cross-checked with an in-house implementation. The counterterm diagram figure $4(\mathrm{~g})$ is constructed by varying the tree-level coupling of the $\chi \chi h_{i}$ vertex $(i=1,2)$

$$
C_{\chi \chi h_{i}}=-\frac{m_{h_{i}}^{2}}{v_{s}} R_{i 2}
$$

yielding

$$
\delta C_{\chi \chi h_{i}}=-\frac{R_{i 2}}{v_{s}} \delta m_{h_{i}}^{2}-\frac{\delta R_{i 2} m_{h_{i}}^{2}}{v_{s}}=-\frac{R_{i 2}}{v_{s}} \delta m_{h_{i}}^{2}-\frac{R_{i 1} m_{h_{i}}^{2}}{v_{s}} \delta \alpha .
$$

Note that, since we are using the standard tadpole scheme, the introduction of a counterterm for the singlet VEV $v_{s}$ is not required to obtain a UV finite result (cf. discussion 

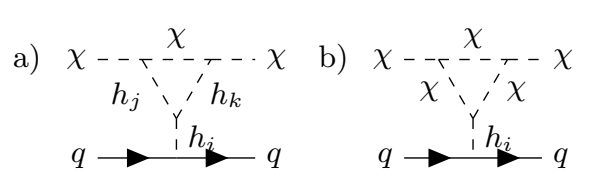

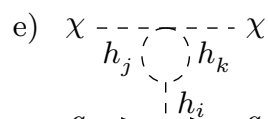

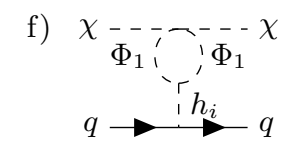

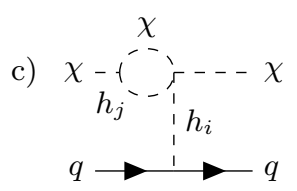
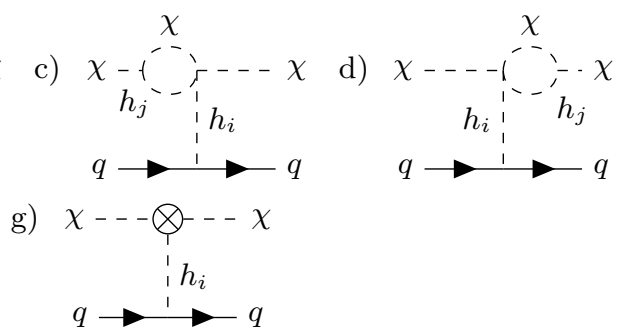

Figure 4. The EW NLO corrections to the upper vertex. The indices $i, j, k=1,2$ indicate the respective Higgs mediator $h_{1}, h_{2}$. The field insertion is given by $\Phi_{1}=\left\{\chi, G^{(0, \pm)}\right\}$.

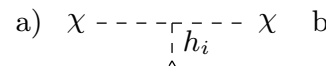

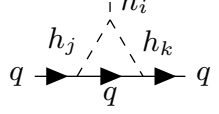

f) $\chi$

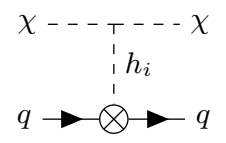

b) $\chi---r_{i}-\chi$

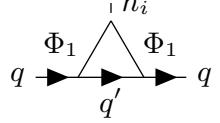

c) $\chi-\cdots---\chi$

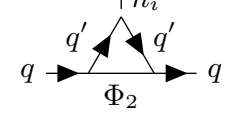

d) $\chi---r-\bar{h}-\chi$
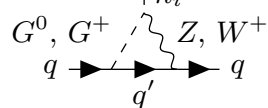

e) $\chi----\ulcorner--\chi$

$Z, W^{+}$今 $G^{0}, G^{+}$

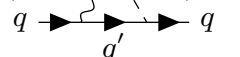

Figure 5. The EW NLO corrections to the lower vertex. The indices $i, j, k=1,2$ indicate the respective Higgs mediator $h_{1}, h_{2}$. The possible field insertions are given by $\Phi_{1}=\left\{G^{(0, \pm)}, Z, W^{ \pm}\right\}$, $\Phi_{2}=\left\{h_{i}, G^{(0, \pm)}, \gamma, Z, W^{ \pm}\right\}$. The quark $q^{\prime}$ corresponds to the up- or down-type quark depending on the field insertion, respectively. Note that for simplicity a diagonal CKM matrix is assumed.

in 3.5). The corresponding counterterm amplitudes read then

$$
\begin{aligned}
& i A_{u p V, h_{1}}^{\mathrm{CT}}=\frac{-C_{q q h_{1}}}{m_{h_{1}}^{2}}\left[\delta C_{\chi \chi h_{1}}+\frac{1}{2}\left(C_{\chi \chi h_{1}} \delta Z_{h_{1} h_{1}}+C_{\chi \chi h_{2}} \delta Z_{h_{2} h_{1}}\right)+C_{\chi \chi h_{1}} \delta Z_{\chi \chi}\right] \bar{u}\left(p_{q}\right) u\left(p_{q}\right), \\
& i A_{u p V, h_{2}}^{\mathrm{CT}}=\frac{-C_{q q h_{2}}}{m_{h_{2}}^{2}}\left[\delta C_{\chi \chi h_{2}}+\frac{1}{2}\left(C_{\chi \chi h_{2}} \delta Z_{h_{2} h_{2}}+C_{\chi \chi h_{1}} \delta Z_{h_{1} h_{2}}\right)+C_{\chi \chi h_{2}} \delta Z_{\chi \chi}\right] \bar{u}\left(p_{q}\right) u\left(p_{q}\right),
\end{aligned}
$$

with the quark Higgs coupling $(i=1,2)$

$$
C_{q q h_{i}}=-\frac{g m_{q}}{2 m_{W}} R_{i 1}
$$

and the quark spinors $u$. The artificially introduced $\delta Z$ factors for the internal Higgs mediator are again included to ensure the proper cancellation in the sum of all topologies.

\subsubsection{Lower vertex (loV) corrections}

In figure 5 all diagrams needed for the lower vertex, in the following referred as $l o V$, are shown. The diagrams (a-e) correspond to the genuine one-loop diagrams calculated for vanishing momentum transfer. The counterterm amplitude figure $5(\mathrm{f})$ is obtained in the same way as for the $u p V$. The tree-level coupling of the lower vertex $q q h_{i}$ is given in eq. (4.14), hence the counterterm for this vertex reads

$$
\delta C_{q q h_{i}}=\frac{-g m_{q}}{2 m_{W}}\left(R_{i 1}\left(-\frac{\delta m_{W}^{2}}{2 m_{W}^{2}}+\frac{\delta g}{g}+\frac{\delta m_{q}}{m_{q}}\right)-R_{i 2} \delta \alpha\right),
$$



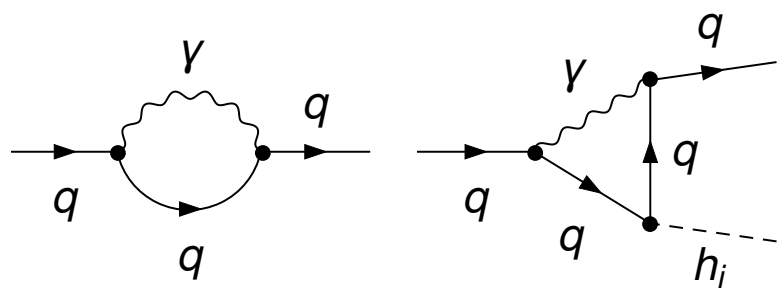

Figure 6. The QED subset. Left: The quark self-energy containing an internal photon line. Right: The vertex correction with an internal photon line.

and the full CT amplitude

$$
\begin{aligned}
& i \mathcal{A}_{l o V, h_{1}}^{C T}=\frac{-C_{\chi \chi h_{1}}}{m_{h_{1}}^{2}}\left(\delta C_{q q h_{1}}+\frac{1}{2}\left(C_{q q h_{1}} \delta Z_{h_{1} h_{1}}+C_{q q h_{2}} \delta Z_{h_{2} h_{1}}+C_{q q h_{1}} \delta Z_{q q}^{L}+C_{q q h_{1}} \delta Z_{q q}^{R}\right)\right), \\
& i \mathcal{A}_{l o V, h_{2}}^{C T}=\frac{-C_{\chi \chi h_{2}}}{m_{h_{2}}^{2}}\left(\delta C_{q q h_{2}}+\frac{1}{2}\left(C_{q q h_{2}} \delta Z_{h_{2} h_{2}}+C_{q q h_{1}} \delta Z_{h_{1} h_{2}}+C_{q q h_{2}} \delta Z_{q q}^{L}+C_{q q h_{2}} \delta Z_{q q}^{R}\right)\right) .
\end{aligned}
$$

The presence of charged particles in the final states indicates additional infrared (IR) divergencies in the amplitudes. The introduction of real radiation to regulate these IR divergencies does not work in this context, since the matching to the parton operators in eq. (4.4) occurs at the amplitude level and the cancellation of the IR divergencies happens at the cross section level. Furthermore, the inclusion of real corrections would also introduce additional tensor structures in the amplitude which have to be accounted for in the parton operator basis.

The IR divergent parts of the amplitude form a closed subset of diagrams referred to as QED subset in the following and all diagrams contain an internal photon line. The corresponding diagrams are depicted in figure 6 where the self-energy of the quarks enters through the mass counterterm $\delta m_{q}$ and the field strength renormalisation constants $\delta Z_{q q}^{L / R}$, and the vertex corrections are part of the genuine one-loop vertex corrections of the lower vertex. One possible solution is the expansion of the QED subset in terms of the external quark momentum $p_{q}$ yielding an IR safe result as discussed in ref. [37]. However, the U(1) symmetry of the potential leads to the complete cancellation of the QED subset, such that no IR divergencies are present in the final renormalized amplitude of the lower vertex corrections. Hence no additional treatment is required to regulate IR divergencies.

\subsubsection{Box diagrams}

The box and triangle topologies contributing to the DM-quark interactions are presented in figure 7 , where the incoming momenta are denoted by $p_{1}$ and $p_{2}$, respectively. For simplicity, the triangle diagrams containing Goldstone bosons $\left(G^{0}, G^{ \pm}\right)$are not shown, but they are included in the calculation and treated in the same way as the Higgs mediator triangle diagrams in figure 7 . The definition of the momenta reflecting the vanishing momentum 


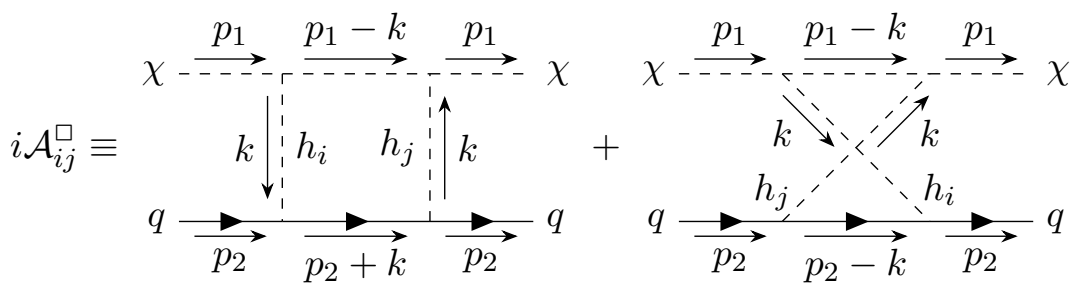

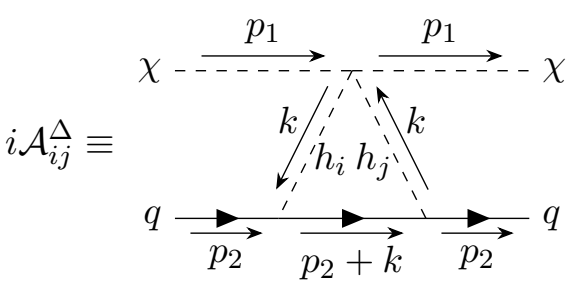

Figure 7. Box topologies contributing to the DM-quark scattering referred to as $\mathcal{A}^{\square}$ and the triangle topologies denoted by $\mathcal{A}^{\Delta}$. The indices $i, j$ denote the Higgs mediators $h_{1}, h_{2}$.

transfer limit allows to express the diagrams as

$$
\begin{aligned}
i \mathcal{A}_{i j}^{\square}= & i^{4} A_{i j} \bar{u}\left(p_{2}\right) \int \frac{d^{4} k}{(2 \pi)^{4}} \frac{1}{k^{2}-m_{i}^{2}} \frac{1}{\left(p_{1}-k\right)^{2}-m_{\chi}^{2}} \frac{1}{k^{2}-m_{j}^{2}} \\
& \cdot\left(\frac{\not p_{2}+\not k+m_{q}}{\left(p_{2}+k\right)^{2}-m_{q}^{2}}+\frac{\not p_{2}-\not k+m_{q}}{\left(p_{2}-k\right)^{2}-m_{q}^{2}}\right) u\left(p_{2}\right), \\
i \mathcal{A}_{i j}^{\Delta}= & i^{4} B_{i j} \bar{u}\left(p_{2}\right) \int \frac{d^{4} k}{(2 \pi)^{4}} \frac{1}{k^{2}-m_{i}^{2}} \frac{1}{k^{2}-m_{j}^{2}} \frac{\not p_{2}+\not k+m_{q}}{\left(p_{2}+k\right)^{2}-m_{q}^{2}} u\left(p_{2}\right),
\end{aligned}
$$

The generic couplings are defined as $A_{i j}=a_{i} a_{j} b_{i} b_{j}$ and $B_{i j}=a_{i} a_{j} b_{i j}$, where $a_{i, j}$ and $b_{i, j}$ are the coefficients of the $h_{i, j} \bar{q} q$ and $h_{i, j} \chi \chi$ vertices, respectively, and $b_{i j}$ is the coefficient of the $h_{i} h_{j} \chi \chi$ vertex. The coefficients are given explicitly by

$$
\begin{aligned}
a_{1} & =-i \frac{m_{q} \cos \alpha}{v}, \quad a_{2}=-i \frac{m_{q} \sin \alpha}{v}, \quad b_{1}=-i \frac{m_{h_{1}}^{2} \sin \alpha}{v_{s}}, \quad b_{2}=-i \frac{m_{h_{2}}^{2} \cos \alpha}{v_{s}} \\
b_{11} & =\frac{\sin \alpha}{4 v v_{s}^{2}}\left(v_{s}\left(m_{h_{2}}^{2}-m_{h_{1}}^{2}\right) \cos ^{3} \alpha+v m_{h_{2}}^{2} \cos ^{2} \alpha \sin \alpha+v m_{h_{1}}^{2} \sin ^{3} \alpha\right) \\
b_{22} & =\frac{\cos \alpha}{4 v v_{s}^{2}}\left(v m_{h_{2}}^{2} \cos ^{3} \alpha+v m_{h_{1}}^{2} \cos \alpha \sin ^{2} \alpha+v_{s}\left(m_{h_{2}}^{2}-m_{h_{1}}^{2}\right) \sin ^{3} \alpha\right) \\
b_{12} & =\frac{\cos \alpha \sin \alpha}{4 v v_{s}^{2}}\left(2 v m_{h_{2}}^{2} \cos ^{2} \alpha+2 v m_{h_{1}}^{2} \sin ^{2} \alpha-v_{s}\left(m_{h_{2}}^{2}-m_{h_{1}}^{2}\right) \sin 3 \alpha\right) .
\end{aligned}
$$

The main contributions to the amplitudes in eq. (4.18) come from the regions close to the poles of the propagators, that is where $k^{2}$ is close to the squared Higgs masses $m_{h_{1}}^{2}$ and $m_{h_{2}}^{2}$ which are of the order of several hundreds to thousands of $\mathrm{GeV}^{2}$. In direct detection experiments, the target nucleus is almost at rest and hence the energy of the nucleons can be approximated by the Fermi energy, which is in the order of $\mathrm{MeV}$. Therefore the approximation $p_{2} \ll k$ is valid in these integrals and the denominators that contain $p_{2}$ can 
be expanded as follows [36, 41],

$$
\frac{1}{\left(p_{2} \pm k\right)^{2}-m_{q}^{2}}=\frac{1}{k^{2} \pm 2 p_{2} \cdot k}=\frac{1}{k^{2}} \mp \frac{2 p_{2} \cdot k}{k^{4}}+\mathcal{O}\left(\left(\frac{p_{2} \cdot k}{k^{2}}\right)^{2}\right)
$$

and using the Dirac equation $\not p u(p)=m_{q} u(p)$ we obtain

$$
\begin{aligned}
& i \mathcal{A}_{i j}^{\square}=i^{4} A_{i j} \bar{u}\left(p_{2}\right) \int \frac{d^{4} k}{(2 \pi)^{4}} \frac{1}{k^{2}-m_{i}^{2}} \frac{1}{\left(p_{1}-k\right)^{2}-m_{\chi}^{2}} \frac{1}{k^{2}-m_{j}^{2}}\left(\frac{4 m_{q}}{k^{2}}+\frac{-4 p_{2} \cdot k}{k^{4}} \not k\right) u\left(p_{2}\right), \\
& i \mathcal{A}_{i j}^{\Delta}=i^{4} B_{i j} \bar{u}\left(p_{2}\right) \int \frac{d^{4} k}{(2 \pi)^{4}} \frac{1}{k^{2}-m_{i}^{2}} \frac{1}{k^{2}-m_{j}^{2}}\left(\frac{1}{k^{2}}-\frac{2 p_{2} \cdot k}{k^{4}}\right)\left(2 m_{q}+\not k\right) u\left(p_{2}\right) .
\end{aligned}
$$

The expanded amplitudes in eq. (4.21) can then be reduced with standard techniques to the Passarino-Veltmann integral basis. Furthermore, we emphasise that the expansion leads to reduced scalar integrals not depending on kinematic variables as $s$ allowing to use the matching procedure to the parton operator basis.

\subsubsection{General mapping to the Wilson coefficients}

All diagrams of the NLO corrections presented in sections 4.2.1 to 4.2.4 have only two independent spinor structures contributing to the SI cross section, namely $\bar{u}\left(p_{2}\right) u\left(p_{2}\right)$ (with the remainder of the amplitude independent of momenta) and terms proportional to $\left(p_{1} \cdot p_{2}\right) \bar{u}\left(p_{2}\right) p_{1} u\left(p_{2}\right)$. Hence, the amplitude can be cast into the following form

$$
i \mathcal{A}=i\left(A \bar{u}\left(p_{2}\right) u\left(p_{2}\right)+B\left(p_{1} \cdot p_{2}\right) \bar{u}\left(p_{2}\right) \not p_{1} u\left(p_{2}\right)\right)
$$

with some momentum-independent constants $A$ and $B$. The definition of the twist-2 operator allows to reformulate

$$
\bar{q} i \partial_{\mu} \gamma_{\nu} q=\mathcal{O}_{\mu \nu}^{q}+i \bar{q}\left(\frac{\partial_{\mu} \gamma_{\nu}-\partial_{\nu} \gamma_{\mu}}{2}+\frac{1}{4} g_{\mu \nu} \not \partial\right) q,
$$

where the asymmetric part does not contribute to the SI cross section and it can therefore be dropped. The resulting amplitude and the coefficients can be mapped to the effective Lagrangian containing the parton operators

$$
\mathcal{L}_{\text {eff }}=\left(\frac{1}{2 m_{q}} A+\frac{1}{8} m_{\chi}^{2} B\right) m_{q} \chi \chi \bar{q} q+\frac{1}{2} B\left(\chi i \partial^{\mu} i \partial^{\nu} \chi\right) \mathcal{O}_{\mu \nu}^{q} .
$$

Identifying the coefficients in eq. (4.24) with the Wilson coefficients in eq. (4.3) yields

$$
\begin{aligned}
C_{S}^{q} & =\frac{1}{2 m_{q}} A+\frac{m_{\chi}^{2}}{8} B, \\
C_{T}^{q} & =\frac{m_{\chi}^{2}}{2} B .
\end{aligned}
$$

By using eq. (4.25) the calculated renormalised amplitude can be mapped to the corresponding Wilson coefficient allowing to determine the SI cross section at NLO. 


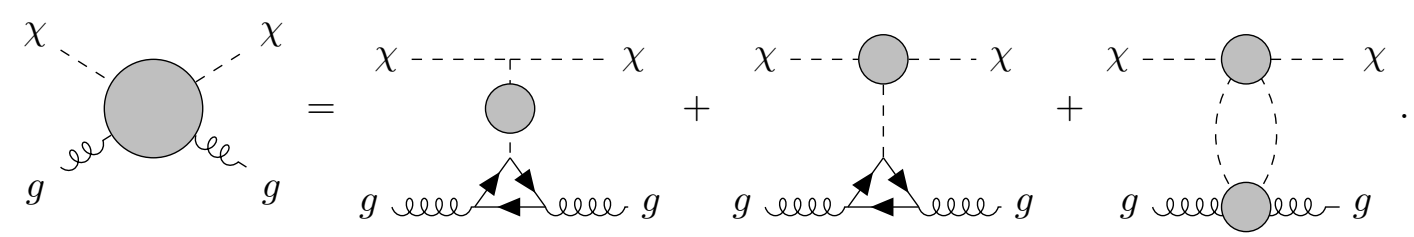

Figure 8. Generic one-loop correction of the DM-gluon interaction. The contributions can be split in mediator, vertex corrections and the effective two-loop contributions. The gray blob indicates the genuine one-loop corrections and the respective counterterm insertion. Figure 9 shows the details of the contribution of each of the blobs of the last diagram.

\subsubsection{Gluon contributions}

Besides the DM-quark interactions also the DM-gluon interactions contribute to the SI cross section. As shown in section 4.1 the leading DM-gluon contributions can be obtained by using the relation between the heavy quark operators and the gluon field strength tensor in eq. (4.8), but this returns a vanishing SI cross section for vanishing momentum transfer $(t \rightarrow 0)$. Therefore, next-to leading order effects have to be taken into account to determine the DM-gluon interactions. The leading non-vanishing gluon interactions are shown in figure 8 which are 2-loop diagrams. The first two diagrams correspond to the generic mediator and upper vertex EW corrections in combination with the effective vertex $g g h_{i}$ which can be calculated in the heavy quark limit (by using eq. (4.8)). The third term corresponds to an effective two-loop calculation which will be discussed later.

The first two diagrams can be calculated using the renormalized upper vertex (section 4.2.2) and the mediator corrections (section 4.2.1) with external quarks instead of the gluon and using eq. (4.8) to effectively determine the DM-gluon interactions. By identifying the gluon parton operator $\mathcal{O}_{s}^{g}$ the respective Wilson coefficient can be deduced in accordance with the quark operators. This method of including the gluon contributions poses several problems, however.

The first problem is that, as will be shown latter, the correct mass dependence, in the limit $m_{\chi} \rightarrow 0$, is not recovered in the limit of zero DM velocity. As discussed in ref. [9] for an exact symmetry the Goldstone boson completely decouples from all of its interactions in the limit of vanishing momentum. Furthermore, it can be shown with the help of a toy model that scattering amplitudes involving Goldstone bosons vanish in the zero-momentum limit although this is not manifest at the Lagrangian level and only occurs through a nontrivial cancellation of terms in the $S$-matrix. The reason is that in the zeromomentum limit the Goldstone state is a symmetry transformation of the ground state and therefore indistinguishable from the vacuum in this limit [9]. The pseudo Goldstone case is similar - we just have to take simultaneously the limit of zero-momentum together with $m_{\chi} \rightarrow 0$ which takes us back to the potential invariant under $\mathrm{U}(1)$.

The second problem is that by this matching, only the diagrams with electroweak corrections to the Higgs boson propagator and the upper DM-Higgs boson vertex can be taken into account. However, electroweak corrections to the lower quark-Higgs boson vertex would obviously interfere with the quark triangle, which makes a matching to heavy 
a)

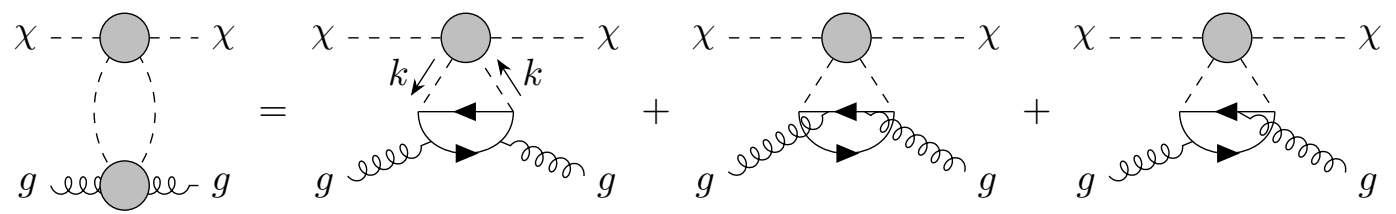

b) $x$

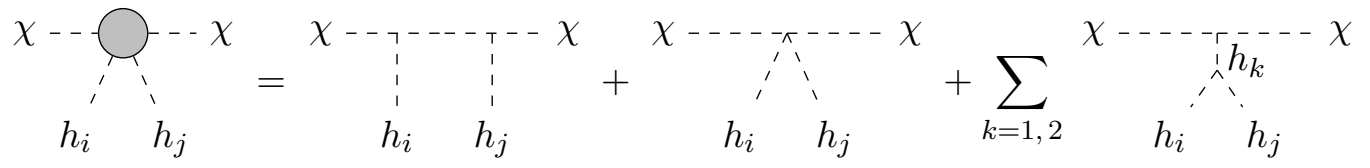

Figure 9. Triangle and box diagrams with external gluons. a) shows the meaning of the lower blob; b) shows the meaning of the upper blob.

quarks non-trivial, since the loops do not factorize. This second problem reveals itself in the framework of our renormalisation scheme in the following two points. By neglecting the lower vertex corrections, the cancellation of the artificially introduced $\delta Z_{h_{i} h_{j}}$ does not occur anymore and an uncanceled finite piece of $\delta Z_{h_{i} h_{j}}$ remains in the Wilson coefficient. In particular, the off-diagonal elements of the wave function renormalisation constants $\delta Z_{h_{i} h_{j}}(i \neq j)$ introduce a mass pole $1 /\left(m_{h_{2}}^{2}-m_{h_{1}}^{2}\right)$ yielding a parametric enhancement for nearly degenerate mass spectra. This divergent enhancement does not correspond to a physical phenomenon but rather to a wrong method for the determination of the DM-gluon interactions. Also, the KOSY scheme for the renormalisation of the mixing counterterm $\delta \alpha$ produces numerically stable (in the sense of no unphysical parametricly enhanced EW NLO corrections or divergencies) NLO predictions if either $\delta \alpha$ and $\delta Z_{h_{i} h_{j}}$ appear in a specific combination or if $\delta \alpha$ appears in a full process several times canceling the mass pole structure [22]. The former occurs e.g. in $1 \rightarrow 2$ Higgs decays yielding a $\delta Z_{h_{i} h_{j}}$ for the on-shell Higgs state and a corresponding $\delta \alpha$ counterterm in the vertex counterterm. The latter is present for instance in the $2 \rightarrow 2$ scattering process $\chi q \rightarrow \chi q$, since $\delta \alpha$ comes both from the upper and from the lower vertices. By neglecting in the lower vertex the EW corrections in the triangle-type diagrams in figure 8 the conditions for a numerically stable KOSY mixing counterterm $\delta \alpha$ are not given, and hence a non-physical enhancement is expected.

The third term in figure 8 corresponds to an effective two-loop calculation, where the two different gray blobs are explained in figure 9. The diagrams in figure 9(a) are calculated using the approach presented in ref. [41] and already applied to the VDM in ref. [29]. Applying the heavy quark limit (valid for mediator masses below the top quark mass) allows us to formulate an effective vertex $h_{i} h_{j} g g$

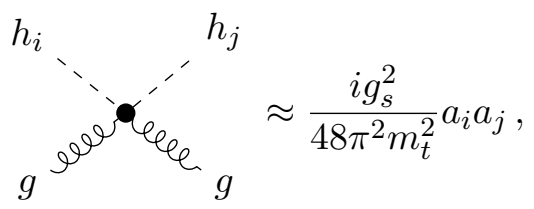

where $a_{i, j}$ are the Higgs-quark couplings defined in eq. (4.19). The vertex is produced by 
the effective Lagrangian

$$
\mathcal{L} \supset \frac{1}{2} \frac{g_{s}^{2}}{48 \pi^{2} m_{t}^{2}} a_{i} a_{j} h_{i} h_{j} G_{\mu \nu}^{a} G^{a \mu \nu},
$$

and therefore the Wilson coefficients $\mathcal{O}_{S}^{g}$ can be extracted by calculating the one-loop diagrams induced by the vertices depicted in figure 9(b). The one-loop corrections induced by the last vertex in figure 9(b) have to be calculated with caution. The first two vertices do not yield a UV pole in the amplitude, hence no counterterm is required. On the other hand, the last vertex generates in general a UV pole requiring a vertex counterterm, since these corrections correspond to an effective vertex correction. However, the U(1) symmetry of the PNGDM ensures the cancellation of all UV poles, yielding a UV safe amplitude.

We emphasise that the inclusion of such effective vertex corrections has to be done with caution, since the cancellation of the UV poles is not guaranteed and is model dependent. Furthermore, these corrections are effective two-loop calculations, where other two-loop contributions are dropped because they are assumed to be small. This is not the case in general. Nevertheless, the size of the included effective two-loop corrections is small compared to the other EW NLO corrections ( $u p V$, lo $V$,med,box) when a scan over the allowed parameter space is performed. Hence, we have included these corrections in our calculation.

In the following we will refer to the inclusion of the EW NLO corrections of the upper vertex or mediator in combination with the effective Higgs-gluon vertex as the approach with the additional gluon contributions. Whereby, the proper SI cross section is calculated solely by taking the effective two-loop contributions into account (third diagram of figure 8). As we will discuss later, these contributions yield only a sub-percentage effect on the overall cross section, hence the inclusion of these contributions does not alter the results significantly.

\section{$5 \quad$ Numerical results}

\subsection{Numerical set-up and parameters}

In the following we list the numerical values used for our study. The SM input parameters are taken as [42]

$$
\begin{aligned}
& m_{u}=0.19 \mathrm{GeV}, \quad m_{c}=1.4 \mathrm{GeV}, \quad m_{t}=172.5 \mathrm{GeV}, \\
& m_{d}=0.19 \mathrm{GeV}, \quad m_{s}=0.19 \mathrm{GeV}, \quad m_{b}=4.75 \mathrm{GeV}, \\
& m_{e}=0.511 \mathrm{MeV}, \quad m_{\mu}=105.658 \mathrm{MeV}, \quad m_{\tau}=1.777 \mathrm{GeV} \text {, } \\
& m_{W}=80.398 \mathrm{GeV}, \quad v=246 \mathrm{GeV}, \\
& m_{Z}=91.188 \mathrm{GeV} \text {. }
\end{aligned}
$$

The SU(2) electroweak gauge coupling $g$ and the Weinberg angle are expressed in terms of the gauge boson masses and the electroweak VEV,

$$
g=2 m_{W} / v=0.653, \quad \sin \theta_{W}=m_{W} / m_{Z}=0.472 .
$$


Note that we chose to renormalize the Higgs sector in the mass-ordered Higgs basis $h_{1}$ and $h_{2}$ with the masses $m_{h_{1}}<m_{h_{2}}$. One of the Higgs bosons is identified as the SM-like Higgs boson with a mass of [43]

$$
m_{h}=125.09 \mathrm{GeV},
$$

and the non-SM like Higgs boson will be referred to as $\phi$, with mass $m_{\phi}$. Both mass hierarchies $m_{h}<m_{\phi}$ and $m_{\phi}<m_{h}$ are allowed in the analysis.

In the following, we refer to the SI cross section as the SI cross section obtained by the scattering on a proton

$$
\sigma \equiv \sigma_{p},
$$

where the proton mass is given by

$$
m_{p}=0.938 \mathrm{GeV} \text {. }
$$

The nuclear matrix elements for the proton needed in eq. (4.6) are [33-35]

$$
\begin{aligned}
& f_{u}^{p}=0.01513, \\
& f_{d}^{p}=0.0191, \\
& f_{s}^{p}=0.0447, \\
& f_{g}^{p}=0.92107, \\
& u^{p}(2)=0.22, \\
& c^{p}(2)=0.019, \\
& \bar{u}^{p}(2)=0.034, \\
& \bar{c}^{p}(2)=0.019, \\
& d^{p}(2)=0.11, \\
& s^{p}(2)=0.026 \text {, } \\
& b^{p}(2)=0.012, \\
& \bar{d}^{p}(2)=0.036, \\
& \bar{s}^{p}(2)=0.026 \text {, }
\end{aligned}
$$

and it should be noted that the uncertainties in the determination of these nuclear matrix elements are not taken into account. For the parameter region scan we implemented the PNGDM in ScannerS $[44,45]$ which is now publicly available. ${ }^{3}$

The points generated using ScannerS have to be in agreement with the most relevant experimental and theoretical constraints. Scanners allows to check that the potential is bounded from below, that there is a global minimum and that perturbative unitarity holds. The SM-like Higgs couplings to the remaining SM particles are all modified by the same factor. Hence, the bound on the signal strength [43] is used to constrain this parameter. There are new contributions to the massive gauge-boson self-energies, $\Pi_{W W}\left(q^{2}\right)$ and $\Pi_{Z Z}\left(q^{2}\right)$. The variables $S, T, U[46,47]$ are used to guarantee agreement with the electroweak precision measurements at the $2 \sigma$ level (for the bounds on the model from perturbativity up to Planck scale see [48]).

The collider bounds from LEP, Tevatron and the LHC are all encoded in HiggsBounds 5.6.0 [49] and HiggsSignals 2.3.1 [50]. Agreement at the 95\% confidence level is asked using the exclusion limits for all available searches for non-standard Higgs bosons, including Higgs invisible decays. The corresponding branching ratios are calculated with AnyHdecay 1.1.0 [45]. This code includes the Higgs decay widths, including the state-of-the art higherorder QCD corrections, for the complex singlet model as obtained from sHDECAY [51]. The

\footnotetext{
${ }^{3}$ The model implementation can be found in ScannerS as CxSMDark.
} 


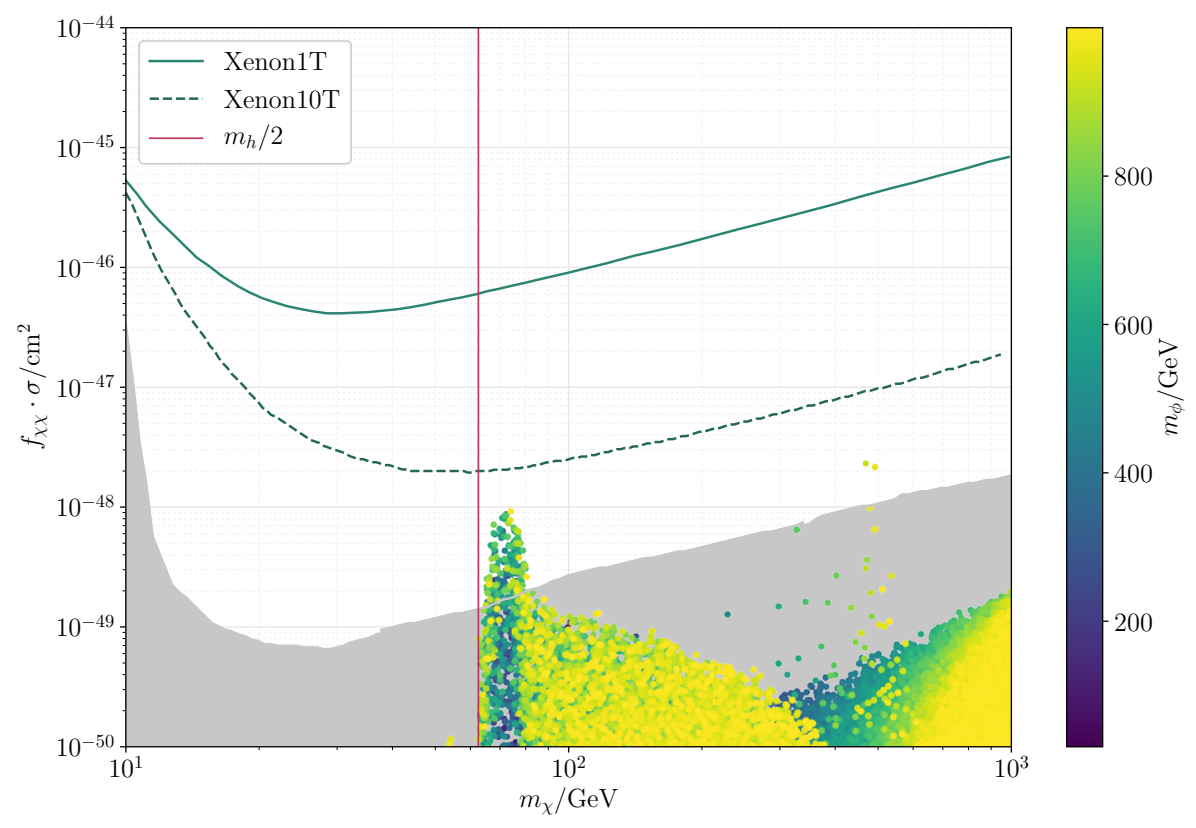

Figure 10. The effective SI-DM nucleon cross section versus the DM mass $m_{\chi}$ is shown, where the color code indicates the value of the non-SM like Higgs boson mass $m_{\phi}$. The gray shaded region denotes the neutrino floor background and the lines the respective (expected) limits of the different experiments. The vertical red line corresponds to $m_{\chi}=m_{h} / 2$.

code sHDECAY is based on the implementation of the singlet models in HDECAY $[52,53]$. For our calculations all EW radiative corrections in HDECAY are turned off for consistency.

The DM relic abundance for each model is calculated with the MicrOMEGAs code [54], which is compared with the current experimental result $\left(\Omega h^{2}\right)_{\mathrm{DM}}^{\mathrm{obs}}=0.1186 \pm 0.002$ from the Planck Collaboration [55]. We do not restrict the DM relic abundance to be exactly at the experimental value but rather that the value predicted by the model has to be equal to or smaller than the observed central value plus $2 \sigma$. This way, we can consider both the dominant and subdominant DM cases simultaneously. Regarding direct detection the XENON1T [56, 57] experiment gives the most stringent upper bound for the DM nucleon scattering.

The scan ranges are chosen to be

$$
\begin{aligned}
& m_{\phi} \in[30 \mathrm{GeV}, 1000 \mathrm{GeV}] \text {, } \\
& m_{\chi} \in[30 \mathrm{GeV}, 1000 \mathrm{GeV}] \text {, } \\
& v_{S} \in[1 \mathrm{GeV}, 1000 \mathrm{GeV}] \text {, } \\
& \alpha \in[\quad-\pi / 2, \quad \pi / 2] \text {. }
\end{aligned}
$$

\subsection{Results and discussion}

We start the discussion with the Xenon plot in figure 10. The effective SI DM-nucleon cross section is shown as a function of the DM mass $m_{\chi}$. Note that the actual SI cross 
section has to be rescaled with the factor

$$
f_{\chi \chi} \equiv \frac{\left(\Omega h^{2}\right)_{\chi}}{\left(\Omega h^{2}\right)_{\mathrm{DM}}}
$$

with the observed relic density $\left(\Omega h^{2}\right)_{\mathrm{DM}}$ and the produced relic density $\left(\Omega h^{2}\right)_{\chi}$ for the DM WIMP $\chi$. As discussed, we do not demand that the DM candidate accounts for the full relic density. When DM is under abundant, the effective factor in eq. (5.8) corrects the cross section accordingly. The relic density is calculated in the standard freeze-out mechanism with the help of MicrOmegas implemented in ScannerS. The color code in figure 10 denotes the value of the non-SM like Higgs boson mass $m_{\phi}$ and the gray shaded region corresponds to the neutrino floor [11]. The different lines correspond to the limits of the different DM detection experiments. The vertical red line indicates the half of the SM-like Higgs boson mass.

All parameter points shown in figure 10 are compatible with the theoretical and experimental constraints described previously. The figure shows that for the entire range of the $\mathrm{DM}$ mass from roughly $50 \mathrm{GeV}$ up to $1 \mathrm{TeV}$, only small mass regions around $m_{\chi} \approx m_{h} / 2$ and $m_{\chi} \approx m_{\phi} / 2$ may yield an effective SI cross section above the neutrino floor. In the case of $m_{\chi} \approx m_{h} / 2$ we can see a large number of points that are basically above but close $m_{h} / 2$; points below $m_{h} / 2$ are excluded by the LHC invisible decay constraints. For the region where $m_{\chi} \approx m_{\phi} / 2$ only a few points for $m_{\phi}$ of the order of $1 \mathrm{TeV}$ are above the neutrino floor. There are however more points in this region that are above the region where most points are concentrated. The fact that only scattered points appear in this region is related to a combination of the experimental constraints. These regions correspond to the two resonances $h$ and $\phi$, respectively. The requirement of proper dark matter abundance leads to the suppression of the coupling between DM and the resonance. However, the kinematical enhancement caused by the resonance compensates for the suppressed couplings that govern DM annihilation in the early Universe. Parameter points below the neutrino floor are not of interest, since these points will not be able to be checked by future direct detection experiments, as the neutrino floor puts a natural limit to the sensitivity of this kind of experiments. The abrupt cut for $m_{\chi}$ below $m_{h} / 2$ is induced by Higgs to invisible searches yielding a strict limit, since in this parameter region the decay $h \rightarrow \chi \chi$ is kinematically allowed. Hence, only a few allowed points are found in this specific parameter region. We emphasise that the tree-level prediction for the SI cross section is zero due to the vanishing momentum-transfer limit, hence the parameter points cannot be constrained by direct detection experiments with tree-level calculations. However, as shown in figure 10, the EW NLO corrections can shift the parameter points above the neutrino floor and approaching the expected limit of the future Xenon 10T experiment. Therefore, the EW NLO corrections might play an important role in the discussion of the sensitivity of the direct detection experiments and derived exclusion limits.

In figure 11 the SI cross section is shown as a function of the non-SM like Higgs boson mass $m_{\phi}$ with the color code indicating the mixing angle $\alpha$. Note that we do not include the factor $f_{\chi \chi}$ here. The SI cross section drops for degenerate neutral Higgs boson masses $\left(m_{\phi} \approx m_{h}\right)$ because the NLO cross section is proportional to $m_{\phi}^{2}-m_{h}^{2}$ as shown in ref. [8]. 

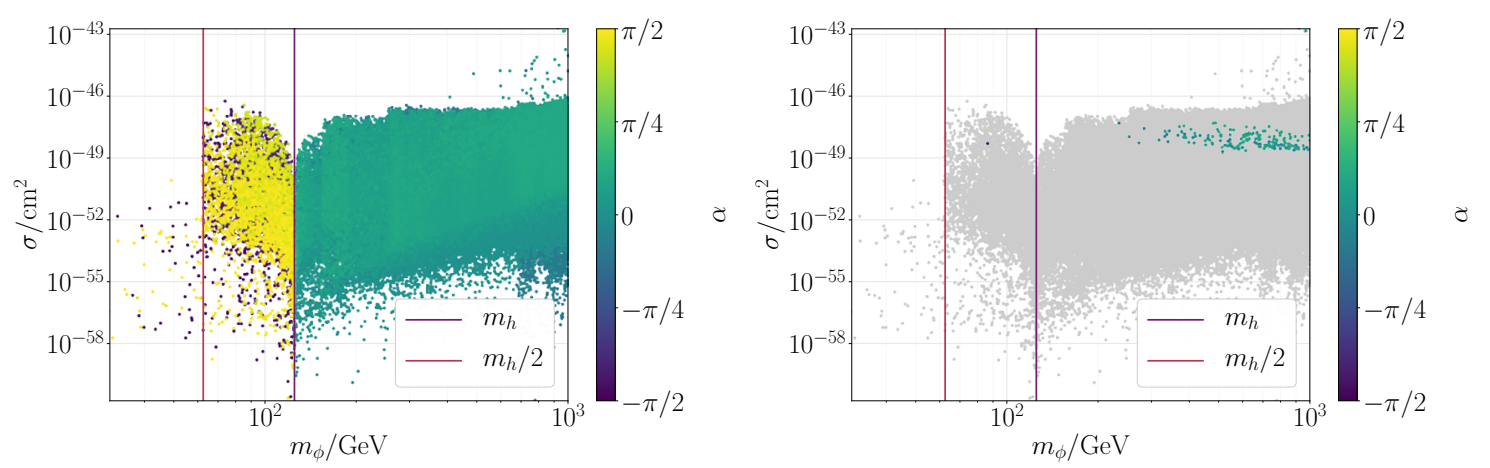

Figure 11. The SI cross section is shown as a function of the non-SM like Higgs boson mass $m_{\phi}$. The gray points denote the full sample passing all experimental and theoretical constraints. The colored points yield SI cross sections above the neutrino floor, where the color code indicates the mixing angle $\alpha$. The two vertical lines indicate $m_{\phi}=m_{h} / 2$ and $m_{\phi}=m_{h}$, respectively. On the left-hand side, all of the about 260.000 parameter points fulfilling the theoretical and experimental constraints are plotted. On the right-hand side, only the parameter points that appear above the neutrino floor are plotted in color and all remaining parameter points are shown in gray.

On the left-hand side, all of the about 260.000 parameter points fulfilling the theoretical and experimental constraints are plotted. On the right-hand side, only the parameter points that lead to direct detection cross sections above the neutrino floor are plotted in color and all remaining parameter points are shown in gray. It is interesting to note that there are allowed points with very large cross sections which, however, do not fulfil the relic density constraints. This way most points with the appropriate relic density have a cross section below $\sim 10^{-46} \mathrm{~cm}^{2}$, except for a few very heavy non-SM like Higgs boson masses. All parameter points with an SI cross section above the neutrino floor have a maximal mixing between the Higgs doublet gauge state $\Phi_{H}$ and the singlet $\Phi_{S}$. Only a single parameter point is above the neutrino floor with one neutral Higgs boson being a singlet-like Higgs boson, meaning that the mass eigenstate is almost given by the singlet field component. This parameter point is also the only parameter point having an inverted Higgs spectrum $\left(m_{\phi}<m_{h}\right)$ while providing an SI cross section above the neutrino floor.

In figure 12 we show the SI cross section as a function of the DM mass $m_{\chi}$, where the DM mass is varied while keeping the other input parameters fixed. On the left side the resulting SI cross section is shown by starting from the benchmark point given in table 1 and then varying only the DM mass while keeping all other parameters fixed, and on the right side we show the results by starting from the benchmark scenario presented in ref. [10] with the input parameters

$$
m_{\phi}=1 \mathrm{TeV}, \quad v_{s}=2 v, \quad \sin \alpha=0.2
$$

and variable DM mass.

The green line corresponds to the SI cross section calculated in the approach presented in section 4.2 and the blue line shows the result for the approach with the additional inclusion of the gluon contributions presented in section 4.2.6. As discussed in section 4.2.6 

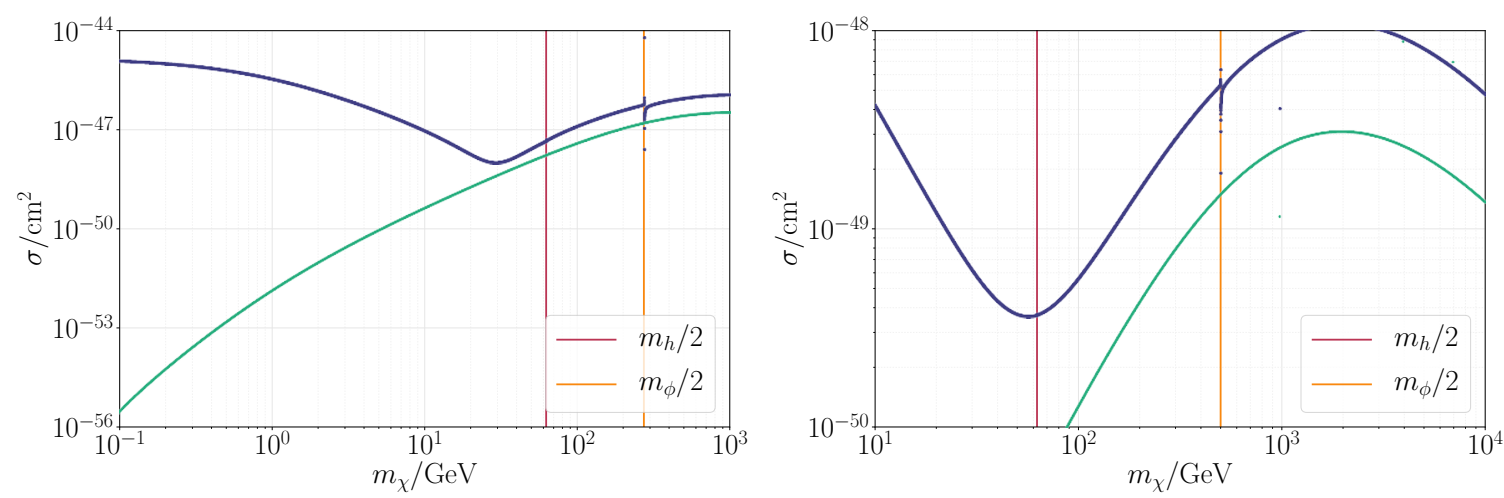

Figure 12. In both figures the SI cross section is shown as a function of the DM mass $m_{\chi}$. The green line indicates the result calculated in our presented approach and the blue line corresponds to the approach including the additional gluon contributions discussed in section 4.2.6. In the left plot, our starting point is the benchmark given in table 1 and on the right side the result using as starting point the benchmark presented in [10] with $m_{\phi}=1 \mathrm{TeV}, v_{s}=2 v$ and $\sin \alpha=0.2$ is shown.

\begin{tabular}{|cccccc|}
\hline$m_{\phi}[\mathrm{GeV}]$ & $m_{\chi}[\mathrm{GeV}]$ & $v_{s}[\mathrm{GeV}]$ & $\alpha$ & $f_{\chi \chi}$ & $\sigma^{(\mathrm{SI})}\left[\mathrm{cm}^{2}\right]$ \\
\hline 546.93 & 72.53 & 152.05 & 0.224 & 0.40 & $8.63 \cdot 10^{-49}$ \\
\hline
\end{tabular}

Table 1. Benchmark point of the PNGDM: The benchmark point is used to illustrate the parameter dependencies in the following. This parameter point provides an SI cross section above the neutrino floor.

the additional gluon contributions induce several problems. The first problem can be clearly seen in both plots in figure 12. The Goldstone nature of the DM candidate $\chi$ requires that the SI cross section scales with the corresponding DM mass $m_{\chi}[8]$, implying that the SI cross section vanishes in the zero DM mass limit, since the Goldstone nature of the DM candidate is restored. Note that this particular behaviour is only expected for vanishing momentum transfer as assumed in the calculation. Our approach (neglecting the gluon contributions) shows for both benchmark points (left and right in figure 12) the desired behaviour for small DM masses $m_{\chi}$ which does not happen when the additional gluon contributions to the SI cross section are included.

As for the second problem related to the approximation performed in the two-loop diagrams, it is not clear how it would reflect on the results. What we can see from the plots is that for large DM masses both approaches yield similar results. The difference is roughly a factor three induced by the inclusion of the gluon contributions. Further, the results presented in ref. [10] are exactly reproduced only if we include the additional gluon contributions. The important point here is to understand that unless a complete 2-loop calculation of the gluon contribution is performed, nothing can be said about the inclusion of approximate calculations of some diagrams.

We calculated all contributing diagrams in the general $R_{\xi}$ gauge in order to be able to check for missing gauge cancellations. As it turned out, our result is completely gauge independent. For the proper cancellation of all gauge dependencies the Goldstone triangle 

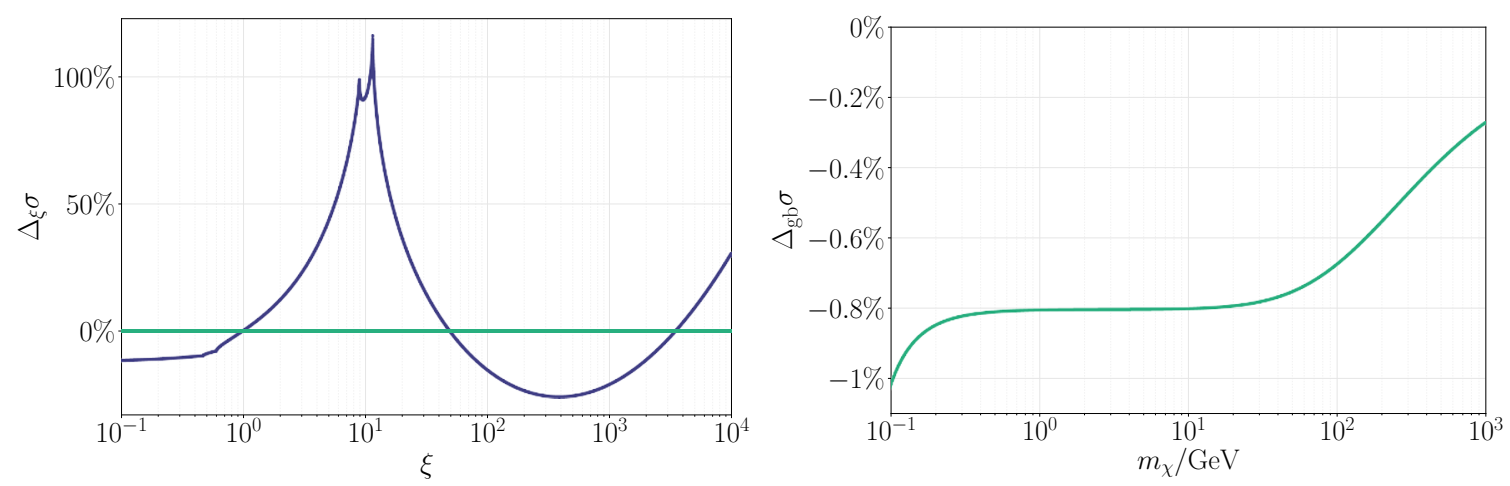

Figure 13. Left: The relative change of the SI cross section as defined in eq. (5.10) is shown as a function of the gauge parameter $\xi$. The green line indicates the result calculated in our presented approach and the blue line corresponds to the approach including the additional gluon contributions discussed in section 4.2.6. Right: The relative change of the SI cross section as defined in eq. (5.11) as a function of the DM mass $m_{\chi}$.

diagrams in figure 7 were crucial. They were needed to properly cancel the gauge dependencies introduced in the vertex corrections. These diagrams are often overseen in the literature. However, the inclusion of the additional gluon contributions introduces gauge dependencies which are not cancelled. We define the relative change

$$
\Delta_{\xi} \sigma \equiv \frac{\sigma-\left.\sigma\right|_{\xi=1}}{\left.\sigma\right|_{\xi=1}}
$$

where $\sigma$ indicates the SI cross section calculated with the additional gluon contributions in the general $R_{\xi}$ gauge and in the Feynman gauge $(\xi=1)$, respectively. In the left plot of figure 13 we show the results for the relative change as a function of the gauge parameter $\xi$. The color scheme follows that of figure 12. Again the benchmark scenario presented in table 1 is used to determine the SI cross section. Obviously, when the additional gluon contributions are included, the variation of the gauge parameter $\xi$ changes the SI cross section significantly preventing to make reasonable predictions for the NLO SI cross section. Hence, not only the correct DM mass dependence is lost with the inclusion of the additional gluon diagrams but also a strong gauge dependence is introduced.

Finally we will discuss the contribution of the third diagram in figure 8 . In the right plot of figure 13 we show the relative difference

$$
\Delta_{\mathrm{gb} \sigma} \equiv \frac{\sigma-\left.\sigma\right|_{\mathrm{nogb}}}{\left.\sigma\right|_{\mathrm{nogb}}},
$$

where $\left.\sigma\right|_{\text {nogb }}$ is the SI cross section calculated without the effective two-loop vertex (third diagram in figure 8) and $\sigma$ the SI cross section as presented. We varied the DM mass $m_{\chi}$ while keeping the other input parameters (same as in table 1) fixed. The most dominant effect of the gluon boxes is obtained for small or large DM masses. Despite that, the overall impact given by the gluon boxes is in the sub-percentage region. Hence, not taking into account the gluon box diagrams and thereby treating all diagrams with external gluons in figure 8 consistently would not significantly alter the overall result. 
In addition to the phenomenological discussion of the SI cross section of the PNGDM we implemented the model in BSMPT $[58,59]$ allowing us to check for a first-order electroweak phase transition in the early universe. For simplicity we force the vacuum expectation value of the DM field component $\chi$ in eq. (2.2) to be equal to zero at all temperatures while determining the global NLO minimum at finite temperature. This way we ensure the stability of the DM candidate and its DM nature. All parameter points above the neutrino floor provide an NLO stable vacuum in the sense that the vacuum ground state of the one-loop effective potential (at zero temperature) is the same as the tree-level ground state. However, all parameter points provide a weak phase transition $v_{c} / T_{c}<1$, where $v_{c}$ is the $\mathrm{SU}(2) \operatorname{VEV}(v)$ at the critical temperature $T_{c}$. The critical temperature is defined as the temperature where the one-loop effective potential has two degenerate minima. A more involved study in the phase structure at finite temperature of the PNGDM might enable a strong first order electroweak phase transition. For instance, allowing the DM field component to evolve a non-zero VEV at finite temperature leads to interesting phenomenological consequences. These studies are left for future work.

\section{Conclusions}

In this work we have calculated the NLO corrections to the spin-independent scattering cross section of a scalar DM particle off a nucleon in a Pseudo Nambu-Goldstone DM model. This model has a scalar potential invariant under a global U(1) symmetry softly broken such that a pseudo Nambu-Goldstone boson originates from the broken symmetry. The cross section was first shown to be proportional to the Dark Matter velocity in ref. [7]. Therefore there was the need to perform the calculation at NLO. There were two independent calculations that appeared very close in time $[8,10]$.

The first calculation [8] was performed by considering from the effective Lagrangian

$$
\mathcal{L}_{\text {eff }}=\sum_{q} C_{S}^{q} \mathcal{O}_{S}^{q}+C_{S}^{g} \mathcal{O}_{S}^{g}+\sum_{q} C_{T}^{q} \mathcal{O}_{T}^{q},
$$

only the first term $\sum_{q} C_{S}^{q} \mathcal{O}_{S}^{q}$. Instead of nuclear matrix elements for the proton an effective Higgs-nucleon coupling was used. Because in this case the one-loop result for the Wilson coefficient is independent of the quark masses, it factorises, and it turns out that the Higgs-nucleon effective coupling is the sum of the nuclear matrix elements. This calculation reproduces the correct dependence of the cross section in the limit of vanishing Dark Matter and is at least $90 \%$ of the total cross section, depending on the parameter points. Hence, relative to this work we have now included the terms $C_{S}^{g} \mathcal{O}_{S}^{g}+\sum_{q} C_{T}^{q} \mathcal{O}_{T}^{q}$.

In the second calculation that appeared in ref. [10] all terms in the effective Lagrangian were used. As previously discussed, the problem in this calculation resides in the gluon diagram contributions (specifically the first two diagrams in figure 8). The 2-loop diagram is not effectively calculated and instead an approximation is performed such that a proper matching between the heavy quarks and the gluon operators cannot be performed. The only way to solve the problem would be to perform the complete 2-loop calculation. The approximation leads therefore to the fact that the Goldstone nature of the DM candidate 
is not recovered and that these gluon contributions present a strong gauge dependence contrary to the rest of the calculation. Therefore these additional gluon contributions should be dropped unless the full two-loop calculation is performed.

It is also worth mentioning that although we have used a different renormalisation scheme than the ones from the two previous calculations our results show a very similar behaviour when the different contributions are compared. Finally, we showed that with the present constraints most of the allowed points are below the neutrino floor and only experiments in the far future will be able to probe them.

\section{Acknowledgments}

We are thankful to Da Huang and M. Spira for fruitful and clarifying discussions. R.S. is supported by the Portuguese Foundation for Science and Technology (FCT), under contracts UIDB/00618/2020, UIDP/00618/2020, PTDC/FIS-PAR/31000/2017, CERN/FISPAR/0002/ 2017 and CERN/FIS-PAR/0014/2019, and by National Science Centre, Poland (NCN) HARMONIA project, contract UMO-2015/18/M/ST2/00518. The work of MM is supported by the BMBF-Project 05H18VKCC1, project number 05H2018.

Open Access. This article is distributed under the terms of the Creative Commons Attribution License (CC-BY 4.0), which permits any use, distribution and reproduction in any medium, provided the original author(s) and source are credited.

\section{References}

[1] V. Silveira and A. Zee, Scalar phantoms, Phys. Lett. B 161 (1985) 136 [InSPIRE].

[2] J. McDonald, Gauge singlet scalars as cold dark matter, Phys. Rev. D 50 (1994) 3637 [hep-ph/0702143] [INSPIRE].

[3] C.P. Burgess, M. Pospelov and T. ter Veldhuis, The minimal model of nonbaryonic dark matter: A singlet scalar, Nucl. Phys. B 619 (2001) 709 [hep-ph/0011335] [INSPIRE].

[4] M.C. Bento, O. Bertolami, R. Rosenfeld and L. Teodoro, Selfinteracting dark matter and invisibly decaying Higgs, Phys. Rev. D 62 (2000) 041302 [astro-ph/0003350] [INSPIRE].

[5] D. Azevedo, M. Duch, B. Grzadkowski, D. Huang, M. Iglicki and R. Santos, Testing scalar versus vector dark matter, Phys. Rev. D 99 (2019) 015017 [arXiv: 1808.01598] [INSPIRE].

[6] C. Arina, A. Beniwal, C. Degrande, J. Heisig and A. Scaffidi, Global fit of pseudo-Nambu-Goldstone Dark Matter, JHEP 04 (2020) 015 [arXiv: 1912. 04008] [INSPIRE].

[7] C. Gross, O. Lebedev and T. Toma, Cancellation Mechanism for Dark-Matter-Nucleon Interaction, Phys. Rev. Lett. 119 (2017) 191801 [arXiv:1708.02253] [INSPIRE].

[8] D. Azevedo, M. Duch, B. Grzadkowski, D. Huang, M. Iglicki and R. Santos, One-loop contribution to dark-matter-nucleon scattering in the pseudo-scalar dark matter model, JHEP 01 (2019) 138 [arXiv: 1810.06105] [inSPIRE].

[9] C.P. Burgess, Goldstone and pseudoGoldstone bosons in nuclear, particle and condensed matter physics, Phys. Rept. 330 (2000) 193 [hep-th/9808176] [INSPIRE].

[10] K. Ishiwata and T. Toma, Probing pseudo Nambu-Goldstone boson dark matter at loop level, JHEP 12 (2018) 089 [arXiv:1810.08139] [INSPIRE]. 
[11] J. Billard, L. Strigari and E. Figueroa-Feliciano, Implication of neutrino backgrounds on the reach of next generation dark matter direct detection experiments, Phys. Rev. D 89 (2014) 023524 [arXiv: 1307.5458] [INSPIRE].

[12] A. Denner, Techniques for calculation of electroweak radiative corrections at the one loop level and results for W physics at LEP-200, Fortsch. Phys. 41 (1993) 307 [arXiv:0709.1075] [INSPIRE].

[13] A. Denner and S. Dittmaier, Electroweak Radiative Corrections for Collider Physics, Phys. Rept. 864 (2020) 1 [arXiv: 1912.06823] [INSPIRE].

[14] F. Bojarski, G. Chalons, D. Lopez-Val and T. Robens, Heavy to light Higgs boson decays at NLO in the Singlet Extension of the Standard Model, JHEP 02 (2016) 147 [arXiv: 1511.08120] [INSPIRE].

[15] M. Krause, R. Lorenz, M. Muhlleitner, R. Santos and H. Ziesche, Gauge-independent Renormalization of the 2-Higgs-Doublet Model, JHEP 09 (2016) 143 [arXiv:1605.04853] [INSPIRE].

[16] A. Denner, L. Jenniches, J.-N. Lang and C. Sturm, Gauge-independent $\overline{M S}$ renormalization in the 2HDM, JHEP 09 (2016) 115 [arXiv: 1607.07352] [INSPIRE].

[17] M. Krause, M. Muhlleitner, R. Santos and H. Ziesche, Higgs-to-Higgs boson decays in a 2HDM at next-to-leading order, Phys. Rev. D 95 (2017) 075019 [arXiv:1609. 04185] [INSPIRE].

[18] M. Krause, D. Lopez-Val, M. Muhlleitner and R. Santos, Gauge-independent Renormalization of the N2HDM, JHEP 12 (2017) 077 [arXiv:1708.01578] [INSPIRE].

[19] L. Altenkamp, S. Dittmaier and H. Rzehak, Renormalization schemes for the Two-Higgs-Doublet Model and applications to $h \rightarrow W W / Z Z \rightarrow 4$ fermions, JHEP 09 (2017) 134 [arXiv: 1704.02645] [INSPIRE].

[20] L. Altenkamp, S. Dittmaier and H. Rzehak, Precision calculations for $h \rightarrow W W / Z Z \rightarrow 4$ fermions in the Two-Higgs-Doublet Model with Prophecy f, JHEP 03 (2018) 110 [arXiv: 1710.07598] [INSPIRE].

[21] M. Fox, W. Grimus and M. Löschner, Renormalization and radiative corrections to masses in a general Yukawa model, Int. J. Mod. Phys. A 33 (2018) 1850019 [arXiv:1705.09589] [INSPIRE].

[22] A. Denner, S. Dittmaier and J.-N. Lang, Renormalization of mixing angles, JHEP 11 (2018) 104 [arXiv: 1808.03466] [INSPIRE].

[23] W. Grimus and M. Löschner, Renormalization of the multi-Higgs-doublet Standard Model and one-loop lepton mass corrections, JHEP 11 (2018) 087 [arXiv:1807.00725] [INSPIRE].

[24] M. Krause, M. Mühlleitner and M. Spira, 2HDECAY - A program for the calculation of electroweak one-loop corrections to Higgs decays in the Two-Higgs-Doublet Model including state-of-the-art QCD corrections, Comput. Phys. Commun. 246 (2020) 106852 [arXiv: 1810.00768] [INSPIRE].

[25] M. Krause and M. Mühlleitner, ewN2HDECAY - A program for the Calculation of Electroweak One-Loop Corrections to Higgs Decays in the Next-to-Minimal Two-Higgs-Doublet Model Including State-of-the-Art QCD Corrections, arXiv:1904.02103 [INSPIRE].

[26] M. Krause and M. Mühlleitner, Impact of Electroweak Corrections on Neutral Higgs Boson Decays in Extended Higgs Sectors, JHEP 04 (2020) 083 [arXiv: 1912.03948] [INSPIRE]. 
[27] A. Pilaftsis, Resonant CP-violation induced by particle mixing in transition amplitudes, Nucl. Phys. B 504 (1997) 61 [hep-ph/9702393] [INSPIRE].

[28] S. Kanemura, Y. Okada, E. Senaha and C.-P. Yuan, Higgs coupling constants as a probe of new physics, Phys. Rev. D 70 (2004) 115002 [hep-ph/0408364] [INSPIRE].

[29] S. Glaus, M. Mühlleitner, J. Müller, S. Patel and R. Santos, Electroweak Corrections to Dark Matter Direct Detection in a Vector Dark Matter Model, JHEP 10 (2019) 152 [arXiv: 1908.09249] [INSPIRE].

[30] M. Sperling, D. Stöckinger and A. Voigt, Renormalization of vacuum expectation values in spontaneously broken gauge theories, JHEP 07 (2013) 132 [arXiv:1305.1548] [INSPIRE].

[31] J. Fleischer and F. Jegerlehner, Radiative Corrections to Higgs Decays in the Extended Weinberg-Salam Model, Phys. Rev. D 23 (1981) 2001 [INSPIRE].

[32] J. Hisano, R. Nagai and N. Nagata, Effective Theories for Dark Matter Nucleon Scattering, JHEP 05 (2015) 037 [arXiv: 1502 .02244] [INSPIRE].

[33] J. Hisano, K. Ishiwata and N. Nagata, Direct Search of Dark Matter in High-Scale Supersymmetry, Phys. Rev. D 87 (2013) 035020 [arXiv:1210.5985] [inSPIRE].

[34] R.D. Young and A.W. Thomas, Octet baryon masses and sigma terms from an $\mathrm{SU}(3)$ chiral extrapolation, Phys. Rev. D 81 (2010) 014503 [arXiv:0901.3310] [INSPIRE].

[35] M.A. Shifman, A.I. Vainshtein and V.I. Zakharov, Remarks on Higgs Boson Interactions with Nucleons, Phys. Lett. B 78 (1978) 443 [INSPIRE].

[36] T. Abe, M. Fujiwara and J. Hisano, Loop corrections to dark matter direct detection in a pseudoscalar mediator dark matter model, JHEP 02 (2019) 028 [arXiv:1810.01039] [INSPIRE].

[37] S. Glaus, M. Mühlleitner, J. Müller, S. Patel and R. Santos, NLO corrections to Vector Dark Matter Direct Detection - An update, PoS CORFU2019 (2020) 046 [arXiv: 2005.11540] [INSPIRE].

[38] A. Denner and S. Dittmaier, Reduction of one loop tensor five point integrals, Nucl. Phys. B 658 (2003) 175 [hep-ph/0212259] [INSPIRE].

[39] A. Denner and S. Dittmaier, Reduction schemes for one-loop tensor integrals, Nucl. Phys. B 734 (2006) 62 [hep-ph/0509141] [INSPIRE].

[40] A. Denner and S. Dittmaier, Scalar one-loop 4-point integrals, Nucl. Phys. B 844 (2011) 199 [arXiv: 1005.2076] [INSPIRE].

[41] F. Ertas and F. Kahlhoefer, Loop-induced direct detection signatures from CP-violating scalar mediators, JHEP 06 (2019) 052 [arXiv: 1902.11070] [INSPIRE].

[42] LHC Higgs Cross Section Working Group collaboration, Handbook of LHC Higgs Cross Sections: 1. Inclusive Observables, arXiv:1101.0593 [INSPIRE].

[43] ATLAS and CMS collaborations, Combined Measurement of the Higgs Boson Mass in pp Collisions at $\sqrt{s}=7$ and $8 \mathrm{TeV}$ with the ATLAS and CMS Experiments, Phys. Rev. Lett. 114 (2015) 191803 [arXiv:1503.07589] [INSPIRE].

[44] R. Coimbra, M.O.P. Sampaio and R. Santos, ScannerS: Constraining the phase diagram of a complex scalar singlet at the LHC, Eur. Phys. J. C 73 (2013) 2428 [arXiv:1301.2599] [INSPIRE].

[45] M. Mühlleitner, M.O.P. Sampaio, R. Santos and J. Wittbrodt, ScannerS: Parameter Scans in Extended Scalar Sectors, arXiv:2007.02985 [INSPIRE]. 
[46] M.E. Peskin and T. Takeuchi, Estimation of oblique electroweak corrections, Phys. Rev. D 46 (1992) 381 [INSPIRE].

[47] W. Grimus, L. Lavoura, O.M. Ogreid and P. Osland, The oblique parameters in multi-Higgs-doublet models, Nucl. Phys. B 801 (2008) 81 [arXiv:0802.4353] [INSPIRE].

[48] A. Falkowski, C. Gross and O. Lebedev, A second Higgs from the Higgs portal, JHEP 05 (2015) 057 [arXiv: 1502.01361] [INSPIRE].

[49] P. Bechtle et al., HiggsBounds-5: Testing Higgs Sectors in the LHC 13 TeV Era, arXiv:2006.06007 [INSPIRE].

[50] P. Bechtle, S. Heinemeyer, O. Stål, T. Stefaniak and G. Weiglein, HiggsSignals: Confronting arbitrary Higgs sectors with measurements at the Tevatron and the LHC, Eur. Phys. J. C $\mathbf{7 4}$ (2014) 2711 [arXiv:1305.1933] [INSPIRE].

[51] R. Costa, M. Mühlleitner, M.O.P. Sampaio and R. Santos, Singlet Extensions of the Standard Model at LHC Run 2: Benchmarks and Comparison with the NMSSM, JHEP 06 (2016) 034 [arXiv: 1512.05355] [inSPIRE].

[52] A. Djouadi, J. Kalinowski and M. Spira, HDECAY: A program for Higgs boson decays in the standard model and its supersymmetric extension, Comput. Phys. Commun. 108 (1998) 56 [hep-ph/9704448] [INSPIRE].

[53] A. Djouadi, J. Kalinowski, M. Muehlleitner and M. Spira, HDECAY: Twenty $y_{+}$years after, Comput. Phys. Commun. 238 (2019) 214 [arXiv:1801.09506] [InSPIRE].

[54] G. Bélanger, F. Boudjema, A. Pukhov and A. Semenov, MicrOMEGAs_3: A program for calculating dark matter observables, Comput. Phys. Commun. 185 (2014) 960 [arXiv: 1305.0237] [INSPIRE].

[55] Planck collaboration, Planck 2015 results. XIII. Cosmological parameters, Astron. Astrophys. 594 (2016) A13 [arXiv:1502.01589] [INSPIRE].

[56] XENON collaboration, First Dark Matter Search Results from the XENON1T Experiment, Phys. Rev. Lett. 119 (2017) 181301 [arXiv:1705.06655] [INSPIRE].

[57] XENON collaboration, Dark Matter Search Results from a One Ton-Year Exposure of XENON1T, Phys. Rev. Lett. 121 (2018) 111302 [arXiv:1805.12562] [INSPIRE].

[58] P. Basler and M. Mühlleitner, BSMPT (Beyond the Standard Model Phase Transitions): A tool for the electroweak phase transition in extended Higgs sectors, Comput. Phys. Commun. 237 (2019) 62 [arXiv: 1803.02846] [INSPIRE].

[59] P. Basler, M. Muhlleitner and J. Müller, BSMPT v2 A Tool for the Electroweak Phase Transition and the Baryon Asymmetry of the Universe in Extended Higgs Sectors, arXiv:2007.01725 [INSPIRE]. 